This paper was retracted on June 7, 2016.

\title{
Dragonbloodin A1 and A2: Flavan Trimers and Anti-inflammatory Principles from Sanguis Draconis
}

\author{
Wen-Ke Du, ${ }^{\dagger}$ Hsin-Yi Hung, ${ }^{\ddagger}$ Ping-Chung Kuo, ${ }^{\ddagger}$ Tsong-Long Hwang, ${ }^{\S}$ Ler-Chun Shiu, ${ }^{\dagger}$ Kom-Bei Shiu, ${ }^{\dagger}$
} E-Jian Lee," Shih-Huang Tai," and Tian-Shung $\mathrm{Wu}^{*}, \neq, \underline{ }$

${ }^{\dagger}$ Department of Chemistry, National Cheng Kung University, 701 Tainan, Taiwan

${ }^{\ddagger}$ School of Pharmacy, National Cheng Kung University Hospital, College of Medicine, National Cheng Kung University, 701 Tainan, Taiwan

${ }^{\S}$ Graduate Institute of Natural Products, College of Medicine, Chang Gung University, Research Center for Industry of Human Ecology and Graduate Institute of Health Industry Technology, Chang Gung University of Science and Technology, Department of Anesthesiology, Chang Gung Memorial Hospital, Taoyuan 333, Taiwan

"Departments of Surgery and Anesthesiology and Institute of Biomedical Engineering, National Cheng Kung University, Medical Center and Medical School, Tainan 701, Taiwan

${ }^{\perp}$ Department of Pharmacy, Tajen University, Pintung 907, Taiwan

Supporting Information

ABSTRACT: Two flavan trimers, dragonbloodin A1 (1) and A2 (2), were isolated as diastereomers from sanguis draconis, a traditional Chinese medicine for regulating blood. The structures of $\mathbf{1}$ and $\mathbf{2}$ were elucidated by spectroscopic analysis and X-ray diffraction. Possible interactions between 1 and 2 were discussed, and possible biosynthesis pathways were deduced. Compounds 1, 2, and their racemic mixture all exhibited inhibition of human neutrophil elastase in a dose-dependent manner.

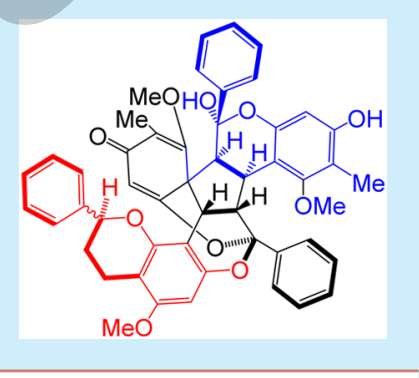

Tnflammation plays a key role in many disease conditions, such 1 as Alzheimer's disease, diabetes, infection, psoriasis, wounds, colitis, arthritis, atherosclerosis, immune diseases, and cancer. ${ }^{1,2}$ Costs associated with inflammatory diseases have resulted in a billion dollar global drug market. Sanguis draconis, also known as dragon's blood, is a deep red resin, an important traditional Chinese medicine (TCM) for blood regulation. ${ }^{3}$ The source of sanguis draconis for TCM use mainly comes from two genuses: Daemonorops (Palmae) in Indonesia, India, and Malaysia and Dracaena (Liliaceae) in Hainan province of China, Vietnam, and Cambodia. ${ }^{3}$ Currently, Daemonorops draco (Willd.) Blume from Indonesia is the most common available species for Chinese medicinal formulas. ${ }^{4}$ The pharmacological studies on Daemonorops draco are in several fields: anticoagulation, ${ }^{5-8}$ antiviral activity, ${ }^{4}$ antibacteria, ${ }^{9}$ anti-inflammatory, ${ }^{10}$ anticancer activity, ${ }^{11-16}$ and osteogenic activity. ${ }^{17}$ So far, the reported chemical constitutions from Daemonorops draco are dracorhodin, nordracorubin, nordracorhodin, dammaradienol, (2S)-5-methoxy-6-methylflavan-7-ol, (2S)-5-methoxyflavan-7-ol, 2,4-dihydroxy-5-methyl-6-methoxychalcone, 2,4-dihydroxy-6-methoxychalcone, dracooxepine, dracoflavans A, B1, B2, C1, C2, D1, and D2, daemonorol series (A-F), abietic acid, and dehydroabietic acid. ${ }^{10,18-23}$ However, studies on the relationship of the chemical constituents and their bioactivities are rare, which drew our attention to investigating novel bioactive compounds from this important TCM. In addition, little progress had been made on the purification of the compounds from dragon's blood through these years, indicating the difficulty and limitation of this field. Our preliminary study showed that the stability of the pure compounds was poor, and many polar bioactive compounds were difficult to purify. However, by means of current technology and chiral columns, two novel bioactive structural complex compounds were isolated and their anti-inflammatory activity was evaluated.

Dragonbloodin A $(\mathbf{1}+\mathbf{2})$ was obtained as colorless needles with $\mathrm{mp}>270{ }^{\circ} \mathrm{C}$. The molecular formula was established as $\mathrm{C}_{50} \mathrm{H}_{44} \mathrm{O}_{10}$ from HR-ESI-MS $\left(m / z 805.3005[\mathrm{M}+\mathrm{H}]^{+}\right.$, calcd 805.3007). But ${ }^{1} \mathrm{H}$ NMR and ${ }^{13} \mathrm{C}$ NMR showed two sets of signals (ratio $=1: 1$ ). An unprecedented skeleton was revealed from X-ray analysis, indicating three flavans with spiro structure. A chiral column (ASTEC CELLULOSE DMP) was used to separate two isomers: dragonbloodin A1 (1) and dragonbloodin A2 (2). ${ }^{4}$

The molecular formula of optically active dragonbloodin A1 (1) was established as $\mathrm{C}_{50} \mathrm{H}_{44} \mathrm{O}_{10}$ from HR-ESI-MS $(\mathrm{m} / \mathrm{z}$ $805.3003[\mathrm{M}+\mathrm{H}]^{+}$, calcd 805.3007 for $\left.\mathrm{C}_{50} \mathrm{H}_{45} \mathrm{O}_{10}\right),[\alpha]^{26}$ +42.4 ( $c 0.09, \mathrm{CHCl}_{3}$ ). The ECD spectrum of 1 showed two positive Cotton effects at 306 and $271 \mathrm{~nm}$ as well as a negative Cotton effect at $249 \mathrm{~nm}) .{ }^{1} \mathrm{H}$ NMR spectrum of 1 in $\mathrm{CDCl}_{3}$ showed the presence of 15 phenyl group protons $\left[\delta_{\mathrm{H}}=7.87(2 \mathrm{H}\right.$,

Received: March 6, 2016 
d, $J=8.2 \mathrm{~Hz}), 7.57(2 \mathrm{H}, \mathrm{t}, J=7.6 \mathrm{~Hz}), 7.48(1 \mathrm{H}, \mathrm{t}, J=7.6 \mathrm{~Hz})$, $7.29-7.30(1 \mathrm{H}, \mathrm{m}), 7.26-7.28(5 \mathrm{H}, \mathrm{m}), 7.24-7.26(2 \mathrm{H}, \mathrm{m})$, and $7.18(2 \mathrm{H}, \mathrm{d}, J=7.6 \mathrm{~Hz})]$, two additional aromatic ring protons $\left[\delta_{\mathrm{H}}=6.35\right.$ and $6.06($ each $\left.1 \mathrm{H}, \mathrm{s})\right]$, an $\alpha$-proton of enone $\left[\delta_{\mathrm{H}}=\right.$ $5.68(1 \mathrm{H}, \mathrm{s})]$, a phenolic $\mathrm{OH}\left[\delta_{\mathrm{H}}=4.93(1 \mathrm{H}, \mathrm{s}, \mathrm{br})\right]$, one $O$ linked proton $\left[\delta_{\mathrm{H}}=4.78(1 \mathrm{H}, \mathrm{dd}, J=10.4,2.4 \mathrm{~Hz})\right]$, four cyclopentane protons $\left[\delta_{\mathrm{H}}=3.96,3.31(\right.$ each $1 \mathrm{H}, \mathrm{d}, J=4.7 \mathrm{~Hz})$, and $3.85,3.72($ each $1 \mathrm{H}, \mathrm{d}, J=9.1 \mathrm{~Hz})$ ], three methoxy groups $\left[\delta_{\mathrm{H}}=3.70,2.94\right.$, and $\left.2.65(\mathrm{each} 3 \mathrm{H}, \mathrm{s})\right]$, a hemiacetal $\mathrm{OH}\left[\delta_{\mathrm{H}}=\right.$ $2.53(1 \mathrm{H}, \mathrm{s}, \mathrm{br})]$, two methylenes $\left[\delta_{\mathrm{H}}=2.61(1 \mathrm{H}, \mathrm{ddd}, J=16.8\right.$, $5.6,3.6 \mathrm{~Hz}), 2.48(1 \mathrm{H}, \mathrm{ddd}, J=17.1,10.9,5.6 \mathrm{~Hz})$, and 2.03, 1.68 (each $1 \mathrm{H}, \mathrm{m})]$, and two methyl groups $\left[\delta_{\mathrm{H}}=2.06\right.$ and 1.23 (each $3 \mathrm{H}, \mathrm{s})]$. Moreover, two methoxy groups $\left(\delta_{\mathrm{H}}=2.94\right.$ and 2.65) and one methyl group $\left(\delta_{\mathrm{H}}=1.23\right)$ are in particular shielded by the spatially close benzene rings. ${ }^{13} \mathrm{C}$ NMR and DEPT 135 spectra of 1 in acetone- $d_{6}$ revealed the presence of 50 carbon signals, including a carbonyl $\left(\delta_{\mathrm{C}}=187.7\right)$, two $O$-linked $\beta$-carbon atoms of enone $\left(\delta_{\mathrm{C}}=171.7\right.$ and 164.2), 30 aromatic ring carbon atoms (O-linked, $C$-linked, unsubstituted, and phenyl groups), two $\alpha$ carbon atoms of enone [methyl-substituted $\left(\delta_{\mathrm{C}}=112.6\right)$ and unsubstituted $\left(\delta_{\mathrm{C}}=106.3\right)$, three $O$-linked carbon atoms [acetal $\left(\delta_{\mathrm{C}}=104.6\right)$, hemiacetal $\left(\delta_{\mathrm{C}}=96.9\right)$, and one tertiary carbon $\left(\delta_{\mathrm{C}}\right.$

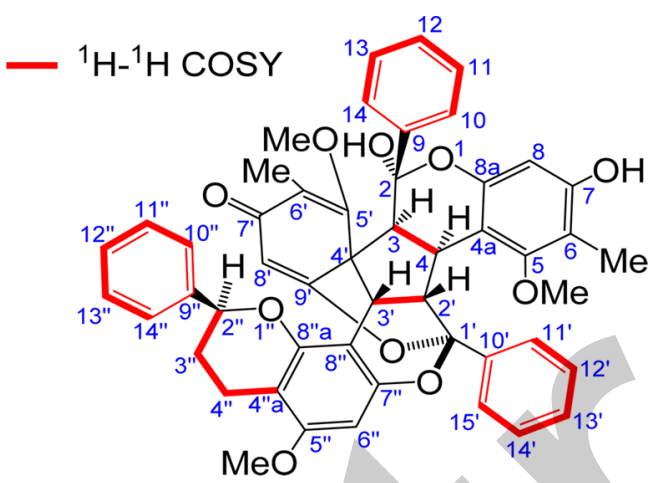

Figure 1. Key ${ }^{1} \mathrm{H}-{ }^{1} \mathrm{H}$ COSY correlations of dragonbloodin $\mathrm{Al}$ (1).

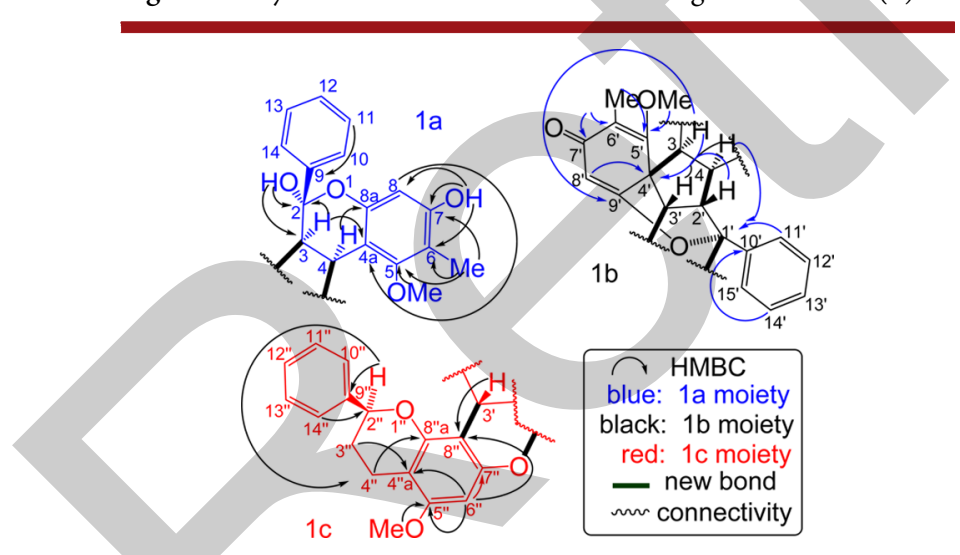

Figure 2. Key HMBC correlations of dragonbloodin A1 (1).

$=96.9)$ ], a spiro carbon atom $\left(\delta_{\mathrm{C}}=54.7\right)$, three methoxy groups $\left(\delta_{\mathrm{C}}=59.2,59.1\right.$, and 55.4), four tertiary carbon atoms of the cyclopentane $\left(\delta_{\mathrm{C}}=51.9,49.2,38.5\right.$, and 35.8$)$, two methylenes $\left(\delta_{\mathrm{C}}=30.0\right.$ and 18.9$)$, as well as two methyl groups $\left(\delta_{\mathrm{C}}=9.4\right.$ and 8.42). On the basis of the analysis of the ${ }^{1} \mathrm{H}-{ }^{1} \mathrm{H}$ COSY, HSQC, and $\mathrm{HMBC}$ spectra, all of the ${ }^{1} \mathrm{H}$ and ${ }^{13} \mathrm{C}$ NMR signals of $\mathbf{1}$ were assigned as shown in Table S1. Dragonbloodin A1 (1) includes three parts: hemiacetal flavan moiety (1a), spiro flavan moiety (1b), and normal flavan moiety (1c).
The ${ }^{1} \mathrm{H}-{ }^{1} \mathrm{H}$ COSY spectrum of $\mathbf{1}$ showed the presence of six spin systems (C3 to $\mathrm{C} 4, \mathrm{C} 2^{\prime}$ to $\mathrm{C}^{\prime}{ }^{\prime}, \mathrm{C} 2^{\prime \prime}$ to $\mathrm{C} 44^{\prime \prime}$, and three phenyl groups; Figure 1). In the $\mathrm{HMBC}$ spectrum, the correlations between $\mathrm{H} 3$ and $\mathrm{C} 2 / \mathrm{C} 4 \mathrm{a}, \mathrm{H} 4$ and $\mathrm{C} 8 \mathrm{a}, \mathrm{H} 8$ and $\mathrm{C} 4 \mathrm{a}, \mathrm{H} 11$ (H13) and C8a, OH-2 and C2/C3, OMe-5 and C5, $\mathrm{Me}-6$ and $\mathrm{C} 5 / \mathrm{C} 6 / \mathrm{C} 7$, as well as between $\mathrm{OH}-7$ and $\mathrm{C} 6 / \mathrm{C} 7 / \mathrm{C} 8$ indicated the presence of a hemiacetal flavan moiety (1a).The correlations between $\mathrm{H} 2^{\prime \prime}$ and $\mathrm{C} 4 " / \mathrm{C} 9 ", \mathrm{H} 3^{\prime \prime}$ and $\mathrm{C} 4{ }^{\prime \prime} \mathrm{a}, \mathrm{H} 4$ " and C8"a, H6" and C4"a/C5"/C7"/C8", H10"/H14" and C2" as well as between OMe-5" and C5" allowed the establishment of a normal flavan moiety (1c). The correlations between $\mathrm{H} 11^{\prime} /$ $\mathrm{H}^{\prime} 5^{\prime}$ and $\mathrm{C}^{\prime}, \mathrm{H} 12^{\prime} / \mathrm{H} 14^{\prime}$ and $\mathrm{C} 10^{\prime}$, OMe-5' and $\mathrm{C}^{\prime}, \mathrm{Me}-6^{\prime}$ and $\mathrm{C}^{\prime} / \mathrm{C}^{\prime} / \mathrm{C}^{\prime}$ and between $\mathrm{H} 3 / \mathrm{H} 4 / \mathrm{H} 2^{\prime} / \mathrm{H}^{\prime} / \mathrm{H}^{\prime}{ }^{\prime}$ and $\mathrm{C}^{\prime}{ }^{\prime}$ were indicated for the spiro flavan moiety $(\mathbf{1 b})$. Moreover, the $\mathrm{HMBC}$ correlations also prove that the hemiacetal flavan moiety (1a) was connected with the spiro flavan moiety (1b) by the correlations between $\mathrm{H} 3$ and $\mathrm{C}^{\prime}$, and $\mathrm{H} 4$ and $\mathrm{Cl}^{\prime}$ ). The spiroflavan moiety (1b) was linked to a normal-flavan moiety (1c) by the correlation between $\mathrm{H}^{\prime}$ and $\mathrm{C}^{\prime \prime}$ (Figure 2).

Dragonbloodin A2 (2) obtained from the cocrystal in chiral system showed the same molecular formula, $\mathrm{C}_{50} \mathrm{H}_{44} \mathrm{O}_{10}$. The ECD spectrum of 2 showed opposite Cotton effects to 1 at 306,

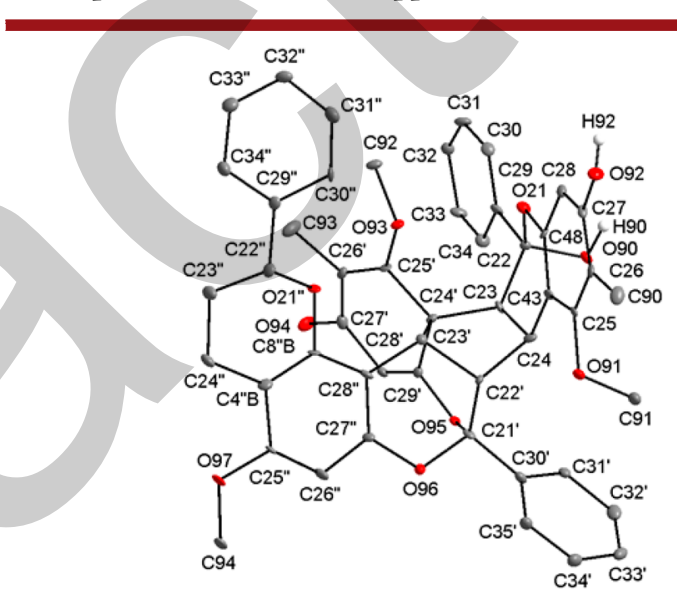

Figure 3. ORTEP diagram of dragonbloodin A1 is presented with thermal ellipsoids for non-hydrogen atoms shown at $15 \%$ probability level.

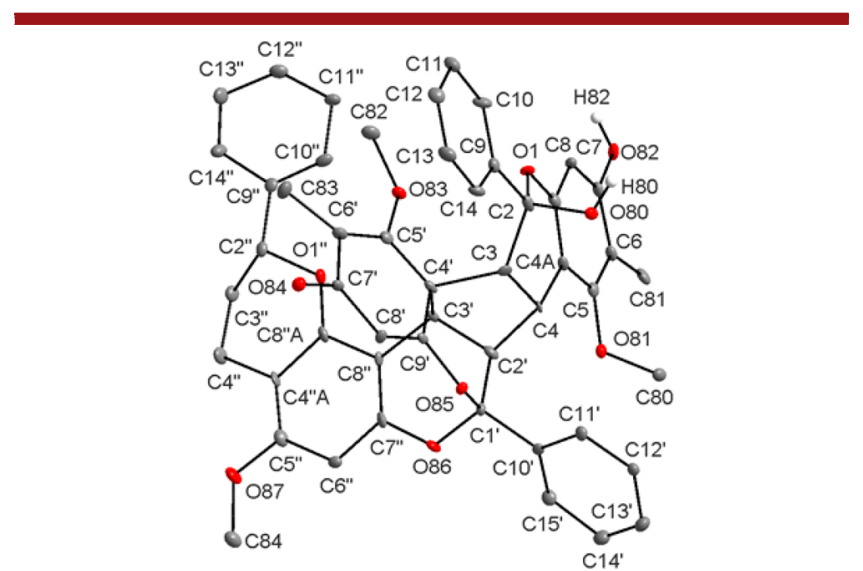

Figure 4. ORTEP diagram of dragonbloodin A1 is presented with thermal ellipsoids for non-hydrogen atoms shown at $15 \%$ probability level.

270 , and $248 \mathrm{~nm}$. The NMR data of 2 (Table S1) were similar to those of 1 except for protons of OMe-5', Me-6', H2", H3", H4", 

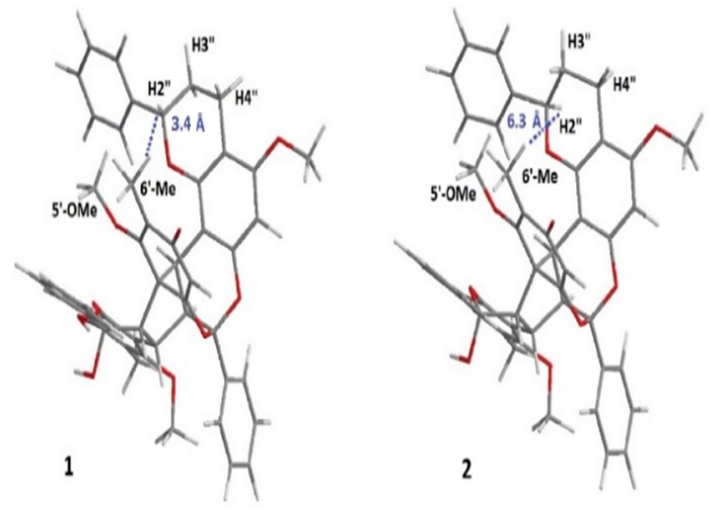

Figure 5. Key differences of the NOESY correlation between $\mathbf{1}$ and $\mathbf{2}$.

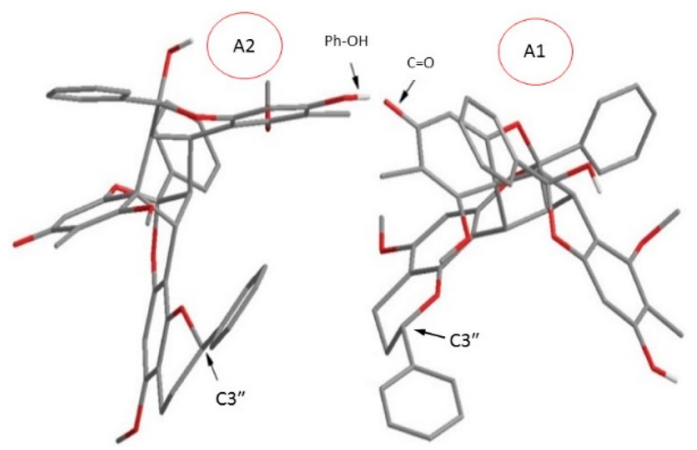

Figure 6. Possible fitting mode between $\mathbf{1}$ and $\mathbf{2}$ via hydrogen bonding and the difference of fitting ability from disordered moieties.

and phenyl group of the normal flavan moiety due to intramolecular shielding effects. The carbon signals were also similar to those of 1 except for C2", C3", and C4" on normal flavan moiety due to different conformation.

The stereochemistry of dragonbloodin A1 (1) and A2 (2) is complicated because there are many permutations and combinations when three flavans integrated. Finally, suitable crystal of dragonbloodin A $(\mathbf{1}+\mathbf{2})$ for single-crystal X-ray diffraction experiment was obtained. The crystal structure can be successfully solved using either $P 2_{1}$ or $P 2_{1} / n$ as the space group. In $P 2_{1}$, a pair of dragonbloodin diastereomers A1 and A2 are found in the asymmetric unit, whereas in $P 2_{1} / n$, the two isomers are merged as one molecule with a carbon atom, which is disordered in two positions at C2" $\mathrm{X}$ and $\mathrm{C} 2$ " $\mathrm{Y}$ with occupancies of 0.616 and 0.384 , respectively (Figure $\mathrm{S} 1$ ). To emphasize that the single crystals we obtained only contain a pair of isomers, the space group of $P 2_{1}$ was used, producing two ORTEPs for isomers $\mathrm{A} 1$ and $\mathrm{A} 2$ in Figures 3 and 4, respectively.

Further, the difference between $\mathbf{1}$ and $\mathbf{2}$ could be identified via NOESY experiments. The NOE correlation between $\mathrm{H} 2$ " and Me- $6^{\prime}$ can be observed in $\mathbf{1}$ not in $\mathbf{2}$ due to closer spatial distance in 1 (Figure 5). In addition, dracoflavan $\mathrm{C}_{2}$, a known flavan dimer, was also isolated by us and its ECD spectrum showed negative Cotton effects at 271,257 , and $226 \mathrm{~nm} .^{10}$ From the combined ECD and NOE data, $2^{\prime \prime} S$ was assigned to 1 . Thus, the deduced stereocenters of 1 are $\left(2 S, 3 S, 4 S, 1^{\prime} R, 2^{\prime} S, 3^{\prime} R, 2^{\prime \prime} S\right)$ and of $\mathbf{2}$ are $\left(2 S, 3 S, 4 S, 1^{\prime} R, 2^{\prime} S, 3^{\prime} R, 2^{\prime \prime} R\right)$. The $2^{\prime \prime} S$ configuration of $\mathbf{1}$ can be further verified by upfield shifting of the Me- $6^{\prime}$ proton signal $\left(\delta_{\mathrm{H}}=1.17\right)$ of 1 due to shilding effects of the phenyl group in normal flavan moiety $\left(\mathrm{Me}-6^{\prime} \delta_{\mathrm{H}}=0.70\right.$ for 2$)$.

Although pure chiral compounds of $\mathbf{1}$ and $\mathbf{2}$ can be obtained, they are very unstable, possibly due to hemiacetal tautomerization in weak acidic or humid conditions (see the Supporting Information). However, the crystal composed of racemic mixture of $\mathbf{1}$ and $\mathbf{2}$ is relatively stable. In order to explain this phenomenon, we proposed a pose to address the interaction between two molecules. Hydrogen bonding was proposed between A1 carbonyl group (C7') and A2 phenolic $\mathrm{OH}(\mathrm{C} 7)$. This pose also imbeds the reactive hemiacetal group, which accounts for its stability (Figure 6).

In this work, compounds 1 and $\mathbf{2}$ represent the first examples of a new class of flavans with a unique spiro and highly stereospecific backbone. The biosynthesis for $\mathbf{1}$ and $\mathbf{2}$ is proposed as shown in Scheme 1. ${ }^{10,24}$ Nucleophilic attack of $\mathbf{4}$ driven by elimination of tertiary alcohol in $\mathbf{5}$ occurred twice to form flavan

\section{Scheme 1. Proposed Biosynthetic Pathway of 1 and 2}

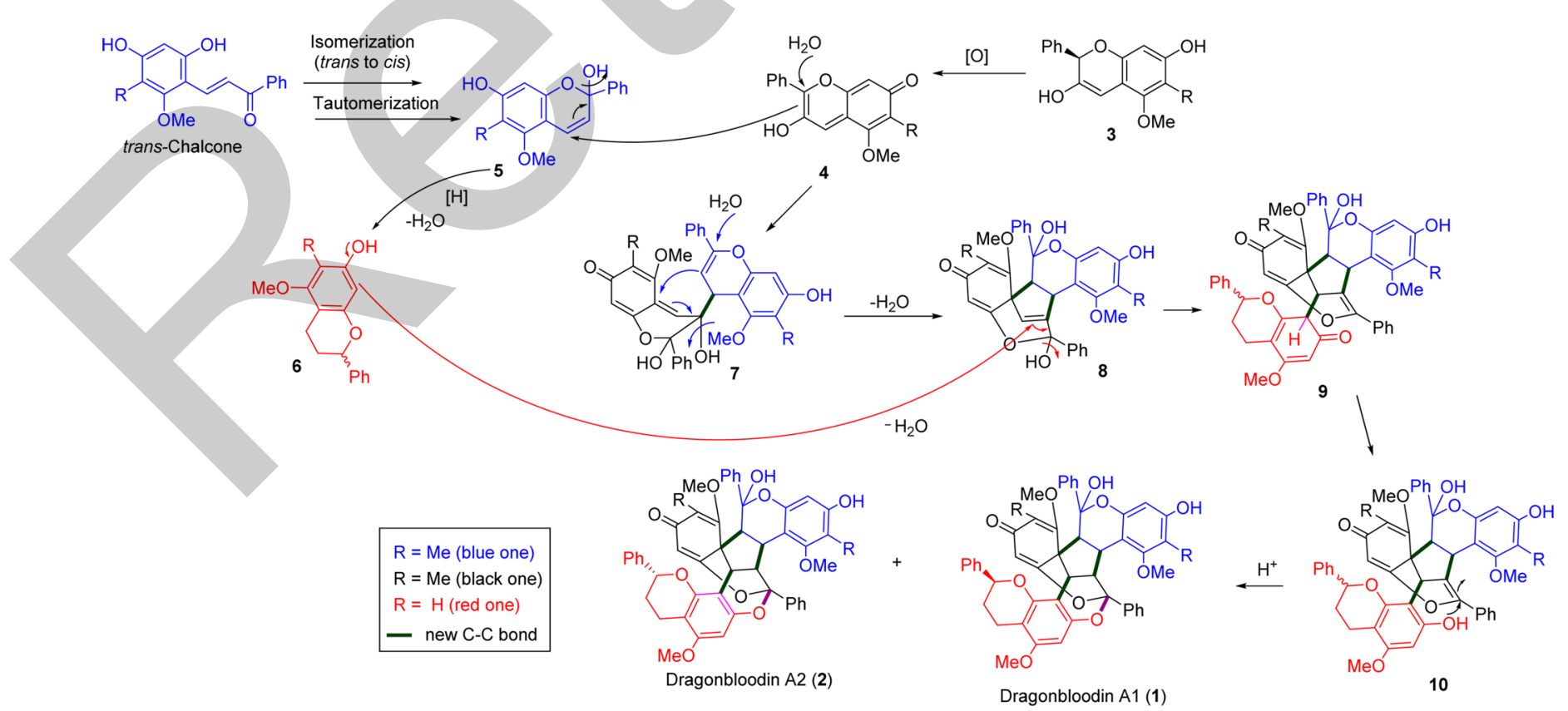


dimer 8. Another flavan $\mathbf{6}$ underwent nucleophilic attack to 8 to form 9 , and a water molecule was eliminated. Aromatization of 9 and final oxygen bridge formation were proposed to give dragonbloodin $\mathrm{A} 1$ and $\mathrm{A} 2$.

Dragonbloodin A $(1+2)$, dragonbloodin A1 (1), and dragonbloodin A2 (2) were evaluated for anti-inflammatory effects on human neutrophil elastase in a cell-free system. $\mathrm{IC}_{50}$ of dragonbloodin A for inhibition of human neutrophil superoxide anion generation and elastase release were $>10$ and $6.53 \mu \mathrm{M}$, respectively. In addition, inhibitions of neutrophil elastase release of dragonbloodin A1 and A2 were seen in a dose-dependent manner from 1 to $10 \mu \mathrm{M}$ (Figures S28 and 29).

\section{ASSOCIATED CONTENT}

\section{(S Supporting Information}

The Supporting Information is available free of charge on the ACS Publications website at DOI: 10.1021/acs.orglett.6b00593.

Experimental methods, spectroscopic data, X-ray analysis of dragonbloodin A, physiochemical data, and biosynthesis pathways of $\mathbf{1}$ and $\mathbf{2}$ (PDF)

$\mathrm{X}$-ray crystallographic data using $P 2_{1}$ as the space group (CIF)

X-ray crystallographic data using $P 2_{1} / n$ as the space group (CIF)

\section{AUTHOR INFORMATION}

\section{Corresponding Author}

*Tel/Fax: +886-6-2747538. E-mail: tswu@mail.ncku.edu.tw. Notes

The authors declare no competing financial interest.

\section{ACKNOWLEDGMENTS}

We are thankful to the Ministry of Science and Technology (MOST 103-2113-M006-001), Taiwan, ROC, and Chang Gung Memorial Hospital (CMRPD1B0481-3, CMRPD1D0281-3, and BMRP450 to H.-L.H.) for financial support of the present research

\section{REFERENCES}

(1) Heneka, M. T.; Carson, M. J.; Khoury, J. E.; Landreth, G. E.; Brosseron, F.; Feinstein, D. L.; Jacobs, A. H.; Wyss-Coray, T.; Vitorica, J.; Ransohoff, R. M.; Herrup, K.; Frautschy, S. A.; Finsen, B.; Brown, G. C.; Verkhratsky, A.; Yamanaka, K.; Koistinaho, J.; Latz, E.; Halle, A.; Petzold, G. C.; Town, T.; Morgan, D.; Shinohara, M. L.; Perry, V. H.; Holmes, C.; Bazan, N. G.; Brooks, D. J.; Hunot, S.; Joseph, B.; Deigendesch, N.; Garaschuk, O.; Boddeke, E.; Dinarello, C. A.; Breitner, J. C.; Cole, G. M.; Golenbock, D. T.; Kummer, M. P. Lancet Neurol. 2015, 14, 388-405.

(2) Pisoschi, A. M.; Pop, A. Eur. J. Med. Chem. 2015, 97, 55-74.

(3) Chinese Herbal Medicine: Materia Medica, 3rd ed.; Bensky, D., Clavey, S., Stoger, E., Eds.; Eastland Press, 1993.

(4) Gupta, D.; Bleakley, B.; Gupta, R. K. J. Ethnopharmacol. 2008, 115, 361-80.

(5) Gibbs, A.; Green, C.; Doctor, V. M. Thromb. Res. 1983, 32, 97-108.

(6) Tsai, W. J.; Hsieh, H. T.; Chen, C. C.; Chen, C. F. J. Chin. Med. $1995,6,59$.

(7) Tsai, W.-J.; Hsieh, H.-T.; Chen, C.-C.; Kuo, Y.-C.; Chen, C.-F. Eur. J. Pharmacol. 1998, 346, 103-110.

(8) Yi, T.; Chen, H.-B.; Zhao, Z.-Z.; Yu, Z.-L.; Jiang, Z.-H. J. Ethnopharmacol. 2011, 133, 796-802.

(9) Rao, G. S. R.; Gerhart, M. A.; Lee, R. T.; Mitscher, L. A.; Drake, S. J. Nat. Prod. 1982, 45, 646-648.
(10) Arnone, A.; Nasini, G.; Vajna de Pava, O.; Merlini, L. J. Nat. Prod. 1997, 60, 971-975.

(11) Xia, M.; Wang, D.; Wang, M.; Tashiro, S.-i.; Onodera, S.; Minami, M.; Ikejima, T. J. Pharmacol. Sci. 2004, 95, 273-283.

(12) Xia, M.; Wang, M.; Tashiro, S.-i.; Onodera, S.; Minami, M.; Ikejima, T. Biol. Pharm. Bull. 2005, 28, 226-232.

(13) Xia, M. Y.; Wang, M. W.; Cui, Z.; Tashiro, S. I.; Onodera, S.; Minami, M.; Ikejima, T. J. Asian Nat. Prod. Res. 2006, 8, 335-343.

(14) He, Y.; Ju, W.; Hao, H.; Liu, Q.; Lv, L.; Zeng, F. J. Huazhong Univ. Sci. Technol., Med. Sci. 2011, 31, 215-219.

(15) Rasul, A.; Ding, C.; Li, X.; Khan, M.; Yi, F.; Ali, M.; Ma, T. Apoptosis 2012, 17, 1104-1119.

(16) Yu, J.-h.; Zheng, G.-b.; Liu, C.-y.; Zhang, L.-y.; Gao, H.-m.; Zhang, Y.-h.; Dai, C.-y.; Huang, L.; Meng, X.-y.; Zhang, W.-y.; Yu, X.-f. Int. J. Med. Sci. 2013, 10, 1149-1156.

(17) Wang, W.; Olson, D.; Cheng, B.; Guo, X.; Wang, K. J Ethnopharmacol. 2012, 142, 168-174.

(18) Robertson, A.; Whalley, W. B. J. Chem. Soc. 1950, 0, 1882-1884.

(19) Nasini, G.; Piozzi, F. Phytochemistry 1981, 20, 514-516.

(20) Nasini, G.; Arnone, A.; Merlini, L. Heterocycles 1989, 29, 11191125.

(21) Arnone, A.; Nasini, G.; Merlini, L. J. Chem. Soc., Perkin Trans. 1 1990, 2637-2640.

(22) Nakashima, K.-i.; Abe, N.; Kamiya, F.; Ito, T.; Oyama, M.; Iinuma, M. Helv. Chim. Acta 2009, 92, 1999-2008.

(23) Shen, C.-C.; Tsai, S.-Y.; Wei, S.-L.; Wang, S.-T.; Shieh, B.-J.; Chen, C.-C. Nat. Prod. Res. 2007, 21, 377-380.

(24) Cardillo, G.; Merlini, L.; Nasini, G.; Salvadori, P. J. Chem. Soc. C 1971, 3967-3970. 


\section{Dragonbloodin A1 and A2 - Flavan Trimers and anti- inflammatory principles from Sanguis Draconis}

Wen-Ke Dư, Hsin-Yi Hung , Ping-Chung Kuo, Tsong-Long Hwang ", Kom-Bei Shiu', E-Jian Lee', Shih-Huang Tai ", Tian-Shung $\mathrm{Wu}^{+,+, *}$

\section{Supporting information}

1. General Experimental Procedure

2. Sanguis Draconis collection and compound isolation process

3. X-ray Crystallographic Analysis of dragonbloodin $\mathrm{A}(\mathbf{1}+\mathbf{2})$

Table S1. A Non-Hydrgoen Atom-to-Atom Contrast Table between dragonbloodin A1 and A2

Table S2. Crystal data, data collection, and refinement parameters

Table S3. Lists of selected bond lengths and angles.

Fig. S1. ORTEP diagram of dragonbloodin A $(\mathbf{1}+\mathbf{2})$

4. Table S4. ${ }^{1} \mathrm{H} \&{ }^{13} \mathrm{C}$ NMR data of $\mathbf{1}$ and $\mathbf{2}$ ( $\delta$ value in ppm, $J$ values in $\mathrm{Hz}$ )

5. Physico-chemical constants of dragonbloodin A1 (1) and A2 (2)

6. Fig. S2. Key ${ }^{1} \mathrm{H}-{ }^{1} \mathrm{H}$ COSY correlations of dragonbloodin $\mathrm{A} 1$ (1)

7. Fig. S3. Key HMBC correlations of dragonbloodin A1 (1)

8. Miscellaneous spectra

Fig. S4. The ECD spectrum of $\mathbf{1}$ and $\mathbf{2}$ had opposite peaks to each other.

Fig. S5. UV spectrum of 1 in $\mathrm{CHCl}_{3}$

Fig. S6. UV spectrum of 2 in $\mathrm{CHCl}_{3}$

Fig. S7. IR spectrum of $\mathbf{1}$ ( $\mathrm{KBr}$ disc)

Fig. S8. IR spectrum of $\mathbf{2}$ (KBr disc)

Fig. S9. HR-ESI-Mass of 1

Fig. S10. HR-ESI-Mass of 2

Fig. S11. ${ }^{1} \mathrm{H}$ NMR spectrum of dragonbloodin $\mathrm{A}(\mathbf{1 + 2})$ in $\mathrm{CDCl}_{3}$

Fig. S12. ${ }^{13} \mathrm{C} \&$ DEPT-135 NMR spectrum of dragonbloodin $\mathrm{A}(\mathbf{1 + 2})$ in $\mathrm{CDCl}_{3}$

Fig. S13. ${ }^{1} \mathrm{H}$ NMR spectrum of 1 in $\mathrm{CDCl}_{3}\left({ }^{1} \mathrm{H}: 700 \mathrm{MHz}\right)$

Fig. S13-1. ${ }^{1} \mathrm{H}$ NMR spectrum of $\mathbf{1}$ in $\mathrm{d}_{6}$-acetone

Fig. S14. ${ }^{13} \mathrm{C} \&$ DEPT-135 NMR spectrum of 1 in $\mathrm{d}_{6}$-acetone $\left({ }^{1} \mathrm{H}: 700 \mathrm{MHz}\right)$

Fig. S15. ${ }^{1} \mathrm{H}-{ }^{1} \mathrm{H}$ COSY spectrum of 1 in $\mathrm{d}_{6}$-Acetone $\left({ }^{1} \mathrm{H}: 700 \mathrm{MHz}\right)$

Fig. S16. HSQC spectrum of 1 in $\mathrm{d}_{6}$-Acetone $\left({ }^{1} \mathrm{H}_{-}{ }^{13} \mathrm{C}: 700 \mathrm{MHz}\right)$

Fig. S17. HMBC spectrum of 1 in $\mathrm{d}_{6}$-Acetone $\left({ }^{1} \mathrm{H}_{-}{ }^{13} \mathrm{C}\right.$ : $\left.700 \mathrm{MHz}\right)$

Fig. S18. Key NOESY spectrum of 1 in $\mathrm{CDCl}_{3}\left({ }^{1} \mathrm{H}-{ }^{1} \mathrm{H}\right.$ : $\left.700 \mathrm{MHz}\right)$

Fig. S19. ${ }^{1} \mathrm{H}$ NMR spectrum of 2 in $\mathrm{CDCl}_{3}\left({ }^{1} \mathrm{H}: 700 \mathrm{MHz}\right)$ 
Fig. S19-1. ${ }^{1} \mathrm{H}$ NMR spectrum of 2 in $\mathrm{d}_{6}$-acetone

Fig. S20. ${ }^{13} \mathrm{C} \&$ DEPT-135 NMR spectrum of 2 in $\mathrm{d}_{6}$-acetone $\left({ }^{1} \mathrm{H}: 700 \mathrm{MHz}\right)$

Fig. S21. ${ }^{1} \mathrm{H}-{ }^{1} \mathrm{H}$ COSY spectrum of 2 in $\mathrm{d}_{6}$-Acetone $\left({ }^{1} \mathrm{H}\right.$ : $\left.700 \mathrm{MHz}\right)$

Fig. S22. HSQC spectrum of 2 in $\mathrm{d}_{6}$-Acetone $\left({ }^{1} \mathrm{H}_{-}{ }^{13} \mathrm{C}: 700 \mathrm{MHz}\right)$

Fig. S23. HMBC spectrum of 2 in $\mathrm{d}_{6}$-Acetone $\left({ }^{1} \mathrm{H}_{-}{ }^{13} \mathrm{C}: 700 \mathrm{MHz}\right)$

Fig. S24. Key NOESY spectrum of 2 in $\mathrm{CDCl}_{3}\left({ }^{1} \mathrm{H}-{ }^{1} \mathrm{H}: 700 \mathrm{MHz}\right)$

Fig. S25. H2"\&6'-Me had NOE (A1, green); had no NOE for A2 (red)

9. Fig. S26. A1 and A2 would transform to other compounds (chiral HPLC)

10. Fig. S27. Biosynthetic pathway of $\mathbf{1}$ and $\mathbf{2}$

11. Fig. S28. Effects of dragonbloodin A1 (1) on the activities human neutrophil elastase in cell-free system.

12. Fig. S29. Effects of dragonbloodin A2 (2) on the activities human neutrophil elastase in cell-free system.

\section{General Experimental Procedure.}

Melting point were determined on Yanaco MP-S3 without temperature corrected. HRESI-MS spectra were acquired from VARIAN ProStar LC/VARIAN 901 FT-ICR Mass and Bruker APEX II FT-Mass. Polarimeter were determined on a JASCO P-2000 Polarimeter with 589nm filter. UV spectra were determined on a HITACHI U-0080-D Spectrophotometer with a $0.1 \mathrm{dm}$ length cell. IR spectra were recorded on a PerkinElmer FT-IR Spectrum RX I using KBr pellets. CD spectra were determined on JASCO J-720 Spectropolarimeter. Semi-preparative HPLC (normal phase) was carried out on an SHIMADZU LC-20AT instrument equipped with UV-VIS detector (SHIMADZU, SPD-10A) and a ASTEC CELLULOSE DMP OD-H column ( $5 \mu \mathrm{m}$, $10.0 \mathrm{~mm}$ I. D. $\times 25 \mathrm{~cm}$; SUPELCO, Sigma-Aldrich). 1D and 2D NMR spectra were recorded on Bruker AVIII 400 and AVIII HD 700 spectrometer (1H: 400 and $700 \mathrm{MHz}$, 13C: 100 and $175 \mathrm{MHz}$ ). X-RAY/SCD were recorded on Bruker SMART APEX II Single-Crystal X-Ray Diffractometer with Mo. TLC analyses were carried out using silica gel $60 \mathrm{~F}_{254}$ (Merck KGaA). Column chromatography was performed on Geduran Si 60 (40-63 $\mu \mathrm{m}$, Merck). All solvents used in column chromatography and HPLC were of pesticide residue analysis grade (Fluka, Sigma-Aldrich), analytical grade (J.T. Baker, AVANTOR) and chromatographic grade (MACRON, AVANTOR and Merck), respectively.

\section{Sanguis Draconis collection and compound isolation process}

The resin of Daemonorops draco was purchased from Chuang Song Zong Pharmaceutical co. Ltd. Taiwan in 2010, and identified by Prof. Chang-Sheng Kuoh, Institute of Biotechnology, National Cheng Kung University. A voucher specimen (Wu- 
2010002) was deposited in Department of Pharmacy, National Cheng Kung University, Taiwan.

The resin of $D$. draco $(3.0 \mathrm{~kg})$ was thoroughly dissolved in $\mathrm{CHCl}_{3}(12.0 \mathrm{~L})$. The insoluble materials were removed by filtration and the filtrate was partitioned with $\mathrm{H}_{2} \mathrm{O}$ $(6.0 \mathrm{~L})$ to yield $\mathrm{CHCl}_{3}$-soluble fraction (about $3.0 \mathrm{~kg}$ ) and water-soluble fraction $(70 \mathrm{~g})$. The $\mathrm{CHCl}_{3}$-soluble extract $(0.5 \mathrm{~kg})$ was subjected to C.C. (Column Chromatography) on a silica gel column with a step gradient ( $n$-hexane-acetone $4: 1,2: 1,1: 1$ and $0: 1$ ) to give ten fractions. Fraction 5 (74.3 g) was separated by C.C. on a silica gel column with a step gradient $\left(\mathrm{CHCl}_{3}\right.$-acetone 49:1, 40:1, 35:1, 29:1, 24:1, 19:1 and 0:1) to give ten fractions: fr. 5-1 (0.1 g), fr. 5-2 (0.1 g), fr. 5-3 (0.2 g), fr. 5-4 (1.6 g), fr. 5-5 (2.2 g), fr. 5-6 (9.6 g), fr. 5-7 (13.2 g), fr. 5-8 (5.4 g), fr. 5-9 (19.3 g) and fr. 5-10 (2.0 g). Fraction 5-7 was separated on a silica gel column with a step gradient (n-hexane-acetone 2:1, $7: 4,3: 2,5: 4,1: 1,1: 2$ and $0: 1)$ to give nine subfractions. Co-crystal of dracoflavan B $(6.1 \mathrm{~g})$ was obtained from subfr. 5-7-3. Dragonbloodin A (303 $\mathrm{mg})$ as a co-crystal was obtained between subfr. 5-7-4 and subfr. 5-7-5. Dragonbloodin A1 and A2 were separated by using a ASTEC Cellulose DMP-OD-H chiral column to yield A1 and A2 with $n$-hexane- $i$-PrOH=70:30 as eluent, flow rate $=2.1 \mathrm{~cm}^{3} \mathrm{~min}^{-1}$, retention time $=16.7$ $\min$ for $\mathrm{A} 2(49.5 \%)$ and $22.1 \mathrm{~min}$ for $\mathrm{A} 1(50.5 \%)$.

X-Ray Crystallography Analysis. Single crystals were grown from $\mathrm{CH}_{2} \mathrm{Cl}_{2} /$ hexane at ambient temperature. Data were collected using a Bruker SMART-CCD diffractometer at $100(2) \mathrm{K}$. The instrument was equipped with graphite-monochromated Mo $\mathrm{K}_{\alpha}$ radiation $(\lambda=0.71073 \AA)$. Empirical absorption corrections were made in light of the results of an azimuthal scan. The crystal structure was determined using the direct method and refined using the full-matrix least-squares method on $\mathrm{F}^{2}$, with all nonhydrogen atoms of the structure refined anisotropically, in SHELXL-97 software. ${ }^{1}$ In the structure refinement, the PLATON-SQUEEZE program was applied for the position-disordered solyent $\left(\mathrm{CH}_{2} \mathrm{Cl}_{2}\right)$ molecules. ${ }^{2}$ All hydrogen atoms were located geometrically with $\mathrm{d}(\mathrm{C}-\mathrm{H})=0.930 \AA$ and $\mathrm{d}(\mathrm{O}-\mathrm{H})=0.820 \AA$. The crystal structure can be successfully solved using either $P 2_{1}$ or $P 2_{1} / n$ as the space group. In $P 2_{1}$, a pair of dragonbloodin diastereomers A1 and A2 are found in the asymmetric unit, whereas in $P 2_{1} / n$, the two isomers are merged as one molecule with a carbon atom, which is disordered in two positions at $\mathrm{C} 2{ }^{\prime \prime} \mathrm{X}$ and $\mathrm{C} 2$ "Y $\mathrm{Y}$ with occupancies of 0.616 and 0.384 , respectively (Fig. S1). To emphasize that the single crystals we obtained only contain a pair of isomers, the space group of $P 2_{1}$ was used, producing two ORTEPs for isomers A1 and A2 in Figs. 1 and 2, respectively. For quick atomic comparison between two 
isomers, the two figures are drawn in a similar orientation with a non-hydrogen atomto-atom contrast table (Table S1). Crystal data of Dragonbloodin A $(1+2)$ was deposited with the Cambridge Crystallographic Data Centre (CCDC 1401169).

\section{References}

1. Sheldrick, G. M. SHELXTL-97; University of Göttingen: Göttingen, Germany.

2. Spek, A. L. J. Appl. Crystallogr. 2003, 36, 7.

Table S1. A Non-Hydrgoen Atom-to-Atom Contrast Table between Dragonbloodin A1 and Dragonbloodin A2

\begin{tabular}{|c|c|c|c|c|c|c|c|}
\hline \multicolumn{3}{|c|}{$\mathrm{A} 1$} & \multicolumn{4}{|c|}{ A2 } \\
\hline C22 & C92 & C33' & C34" & C2 & C82 & C13' & C14" \\
\hline C23 & C93 & C34' & C43 & C3 & C83 & C14' & C4A \\
\hline C24 & C94 & C35' & C48 & C4 & C84 & C15' & C8A \\
\hline C25 & C21' & C22" & C4"B & C5 & C1' & C2" & C4"A \\
\hline C26 & C22' & C23" & C8"B & C6 & C2' & C3" & C8'A \\
\hline C27 & C23' & C24" & O21 & C7 & C3' & C4" & O1 \\
\hline C28 & C24' & C25" & O21" & C8 & C4' & C5'" & O1" \\
\hline C29 & C25' & C26" & O90 & C9 & C5' & C6" & O80 \\
\hline C30 & C26' & C27" & O91 & C10 & C6' & C7" & O81 \\
\hline C31 & C27' & C28" & O92 & C11 & C7' & C8" & O82 \\
\hline C32 & C28' & C29" & O93 & C12 & C8' & C9" & O83 \\
\hline C33 & C29' & C30" & O94 & C13 & C9' & C10" & O84 \\
\hline C34 & C30' & C31" & O95 & C14 & C10' & C11" & O85 \\
\hline C90 & C31' & C32" & O96 & C80 & C11' & C12" & O86 \\
\hline C91 & C32' & C33" & O97 & C81 & C12' & C13" & O87 \\
\hline
\end{tabular}

Table S2. Crystal data

formula

fw $\left(\mathrm{g} \mathrm{mol}^{-1}\right)$

cryst size $\left(\mathrm{mm}^{3}\right)$

cryst syst

space group

$a(\AA)$

$b(\AA)$

$c(\AA)$

$\alpha(\operatorname{deg})$

$\beta$ (deg)
$\mathrm{C}_{50} \mathrm{H}_{44} \mathrm{O}_{10}$

804.85

$0.65 \times 0.43 \times 0.31$

monoclinic

$P 2_{1}$

15.708(2)

17.744(3)

16.623(3)

90

99.335(2) 


$\begin{array}{ll}\gamma(\mathrm{deg}) & 90 \\ V\left(\AA^{3}\right) & 4572.2(12) \\ Z & 4 \\ D_{\text {calc }}\left(\mathrm{g} \mathrm{cm}^{-3}\right) & 1.168 \\ \mu,\left(\mathrm{mm}^{-1}\right) & 0.081 \\ \mathrm{~F}(000) & 1692.0 \\ \text { reflns collected, unique } & 21019 / 13848 \\ R_{l} / w R_{2}(\mathrm{I}>2 \sigma(\mathrm{I})) & 0.0696 / 0.1821 \\ \mathrm{GOF} \text { on } \mathrm{F}^{2} & 1.086 \\ \text { largest diff peak and hole }\left(\mathrm{e} \AA^{-3}\right) & 0.392 /-0.289\end{array}$

Table S3. Lists of selected bond lengths and angles for Dragonbloodin A1 and Dragonbloodin A2

\begin{tabular}{|c|c|c|c|}
\hline (a) Bong Lengths $(\AA)$ : & & & \\
\hline $\mathrm{C} 2-\mathrm{O} 1$ & $1.420(6)$ & $\mathrm{C} 11 "-\mathrm{C} 12^{\prime \prime}$ & $1.345(7)$ \\
\hline $\mathrm{C} 2-\mathrm{O} 80$ & $1.431(6)$ & $\mathrm{C} 12^{\prime \prime}-\mathrm{C} 13^{\prime \prime}$ & $1.379(7)$ \\
\hline $\mathrm{C} 2-\mathrm{C} 9$ & $1.516(7)$ & $\mathrm{C} 13 "-\mathrm{C} 14 "$ & $1.316(8)$ \\
\hline $\mathrm{C} 2-\mathrm{C} 3$ & $1.541(6)$ & $\mathrm{C} 22^{\prime \prime}-\mathrm{O} 21^{\prime \prime}$ & $1.448(4)$ \\
\hline $\mathrm{C} 3-\mathrm{C} 4$ & $1.520(6)$ & C22"-C23" & $1.478(6)$ \\
\hline $\mathrm{C} 3-\mathrm{C} 4{ }^{\prime}$ & $1.650(7)$ & C22"-C29" & $1.513(6)$ \\
\hline $\mathrm{C} 4-\mathrm{C} 2{ }^{\prime}$ & $1.518(6)$ & $\mathrm{C} 23 "-\mathrm{C} 24^{\prime \prime}$ & $1.516(7)$ \\
\hline $\mathrm{C} 4-\mathrm{C} 4 \mathrm{~A}$ & $1.551(6)$ & $\mathrm{C} 24 "-\mathrm{C} 4{ }^{\prime \prime} \mathrm{B}$ & $1.500(7)$ \\
\hline $\mathrm{C} 5-\mathrm{O} 81$ & $1.333(6)$ & $\mathrm{C} 25 "-\mathrm{C} 26 "$ & $1.335(7)$ \\
\hline $\mathrm{C} 5-\mathrm{C} 6$ & $1.368(7)$ & C25"-O97 & $1.393(6)$ \\
\hline $\mathrm{C} 5-\mathrm{C} 4 \mathrm{~A}$ & $1.431(7)$ & C25"-C4"B & $1.469(7)$ \\
\hline C6- - 7 & $1.407(7)$ & $\mathrm{C} 26 "-\mathrm{C} 27^{\prime \prime}$ & $1.378(6)$ \\
\hline C6 - C81 & $1.506(7)$ & C27"-O96 & $1.409(5)$ \\
\hline $\mathrm{C} 7-\mathrm{C} 8$ & $1.371(7)$ & $\mathrm{C} 27^{\prime \prime}-\mathrm{C} 28 "$ & $1.412(6)$ \\
\hline $\mathrm{C} 7-\mathrm{O} 82$ & $1.416(6)$ & C28"- & $1.354(7)$ \\
\hline $\mathrm{C} 8-\mathrm{C} 8 \mathrm{~A}$ & $1.373(7)$ & $\mathrm{C} 28^{\prime \prime}-\mathrm{C} 23^{\prime}$ & $1.496(6)$ \\
\hline $\mathrm{C} 9-\mathrm{C} 14$ & $1.350(7)$ & C29"-C34" & $1.387(7)$ \\
\hline C9- $\mathrm{C} 10$ & $1.387(7)$ & $\mathrm{C} 29 "-\mathrm{C} 30 "$ & $1.404(6)$ \\
\hline $\mathrm{C} 10-\mathrm{C} 11$ & $1.406(7)$ & C30"-C31" & $1.344(8)$ \\
\hline $\mathrm{C} 11-\mathrm{C} 12$ & $1.458(7)$ & C31"-C32" & $1.388(7)$ \\
\hline $\mathrm{C} 12-\mathrm{C} 13$ & $1.293(7)$ & C32"-C33" & $1.356(8)$ \\
\hline $\mathrm{C} 13-\mathrm{C} 14$ & $1.398(7)$ & C33"-C34" & $1.443(8)$ \\
\hline $\mathrm{C} 22-\mathrm{O} 21$ & $1.425(6)$ & $\mathrm{C} 4 " \mathrm{~A}-\mathrm{C} 8 \mathrm{~A}$ & $1.446(7)$ \\
\hline
\end{tabular}




\begin{tabular}{|c|c|c|c|}
\hline $\mathrm{C} 22-\mathrm{O} 90$ & $1.432(4)$ & $\mathrm{C} 8 " \mathrm{~A}-\mathrm{O} 1 "$ & $1.273(6)$ \\
\hline $\mathrm{C} 22-\mathrm{C} 23$ & $1.508(7)$ & $\mathrm{C} 4 " \mathrm{~B}-\mathrm{C} 8{ }^{\prime \prime} \mathrm{B}$ & $1.349(6)$ \\
\hline $\mathrm{C} 22-\mathrm{C} 29$ & $1.537(6)$ & $\mathrm{C} 8 \mathrm{~B}-\mathrm{O} 21 "$ & $1.447(5)$ \\
\hline $\mathrm{C} 23-\mathrm{C} 24^{\prime}$ & $1.557(6)$ & $\mathrm{C} 1{ }^{\prime}-\mathrm{O} 86$ & $1.394(5)$ \\
\hline $\mathrm{C} 23-\mathrm{C} 24$ & $1.572(7)$ & $\mathrm{C} 1{ }^{\prime}-\mathrm{O} 85$ & $1.429(4)$ \\
\hline $\mathrm{C} 24-\mathrm{C} 43$ & $1.472(7)$ & $\mathrm{C} 1^{\prime}-\mathrm{C} 10^{\prime}$ & $1.515(7)$ \\
\hline $\mathrm{C} 24-\mathrm{C} 22^{\prime}$ & $1.566(6)$ & $\mathrm{C} 11^{\prime}-\mathrm{C} 2^{\prime}$ & $1.575(7)$ \\
\hline $\mathrm{C} 25-\mathrm{C} 43$ & $1.390(7)$ & $\mathrm{C} 2^{\prime}-\mathrm{C} 3^{\prime}$ & $1.476(7)$ \\
\hline $\mathrm{C} 25-\mathrm{C} 26$ & $1.416(7)$ & $\mathrm{C} 3^{\prime}-\mathrm{C} 4^{\prime}$ & $1.568(7)$ \\
\hline $\mathrm{C} 25-\mathrm{O} 91$ & $1.423(6)$ & $\mathrm{C} 4^{\prime}-\mathrm{C} 5^{\prime}$ & $1.427(7)$ \\
\hline $\mathrm{C} 26-\mathrm{C} 27$ & $1.366(7)$ & $\mathrm{C} 4^{\prime}-\mathrm{C} 9^{\prime}$ & $1.493(8)$ \\
\hline $\mathrm{C} 26-\mathrm{C} 90$ & $1.503(7)$ & $\mathrm{C} 5^{\prime}-\mathrm{O} 83$ & $1.357(6)$ \\
\hline $\mathrm{C} 27-\mathrm{O} 92$ & $1.331(6)$ & $\mathrm{C} 5^{\prime}-\mathrm{C} 6^{\prime}$ & $1.376(7)$ \\
\hline $\mathrm{C} 27-\mathrm{C} 28$ & $1.399(7)$ & $\mathrm{C} 6^{\prime}-\mathrm{C}^{\prime}$ & $1.523(7)$ \\
\hline $\mathrm{C} 28-\mathrm{C} 48$ & $1.395(7)$ & $\mathrm{C}^{\prime}-\mathrm{O} 84$ & $1.205(6)$ \\
\hline $\mathrm{C} 29-\mathrm{C} 30$ & $1.364(7)$ & $\mathrm{C} 7{ }^{\prime}-\mathrm{C} 8^{\prime}$ & $1.444(7)$ \\
\hline $\mathrm{C} 29-\mathrm{C} 34$ & $1.412(7)$ & $\mathrm{C} 8^{\prime}-\mathrm{C} 9^{\prime}$ & $1.341(7)$ \\
\hline $\mathrm{C} 30-\mathrm{C} 31$ & $1.386(7)$ & C9'-O85 & $1.367(6)$ \\
\hline $\mathrm{C} 31-\mathrm{C} 32$ & $1.321(7)$ & $\mathrm{C} 10^{\prime}-\mathrm{C} 15^{\prime}$ & $1.331(7)$ \\
\hline $\mathrm{C} 32-\mathrm{C} 33$ & $1.452(7)$ & $\mathrm{C} 10^{\prime}-\mathrm{C} 11^{\prime}$ & $1.407(6)$ \\
\hline C33-C34 & $1.362(7)$ & $\mathrm{C} 11^{\prime}-\mathrm{C} 12^{\prime}$ & $1.369(7)$ \\
\hline $\mathrm{C} 43-\mathrm{C} 48$ & $1.389(7)$ & $\mathrm{C} 12^{\prime}-\mathrm{C} 13^{\prime}$ & $1.420(7)$ \\
\hline $\mathrm{C} 48-\mathrm{O} 21$ & $1.387(6)$ & $\mathrm{C} 13^{\prime}-\mathrm{C} 14^{\prime}$ & $1.332(8)$ \\
\hline $\mathrm{C} 80-\mathrm{O} 81$ & $1.446(6)$ & $\mathrm{C} 14^{\prime}-\mathrm{C} 15^{\prime}$ & $1.439(8)$ \\
\hline $\mathrm{C} 82-\mathrm{O} 83$ & $1.431(6)$ & $\mathrm{C} 21^{\prime}-\mathrm{O} 96$ & $1.440(5)$ \\
\hline C83-C6' & $1.522(9)$ & $\mathrm{C} 21^{\prime}-\mathrm{O} 95$ & $1.447(6)$ \\
\hline $\mathrm{C} 84-\mathrm{O} 87$ & $1.413(6)$ & $\mathrm{C} 21^{\prime}-\mathrm{C} 22^{\prime}$ & $1.485(5)$ \\
\hline $\mathrm{C} 91-\mathrm{O} 91$ & $1.415(6)$ & $\mathrm{C} 21^{\prime}-\mathrm{C} 30^{\prime}$ & $1.507(7)$ \\
\hline C92-O93 & $1.441(6)$ & $\mathrm{C} 22^{\prime}-\mathrm{C} 23^{\prime}$ & $1.578(6)$ \\
\hline $\mathrm{C} 93-\mathrm{C} 26^{\prime}$ & $1.515(8)$ & $\mathrm{C} 23^{\prime}-\mathrm{C} 24^{\prime}$ & $1.561(6)$ \\
\hline $\mathrm{C} 94-\mathrm{O} 97$ & $1.447(6)$ & $\mathrm{C} 24^{\prime}-\mathrm{C} 29^{\prime}$ & $1.501(8)$ \\
\hline C2"-O1" & $1.460(5)$ & $\mathrm{C} 24^{\prime}-\mathrm{C} 25^{\prime}$ & $1.569(7)$ \\
\hline C2"-C9" & $1.507(6)$ & $\mathrm{C} 25^{\prime}-\mathrm{C} 26^{\prime}$ & $1.339(7)$ \\
\hline $\mathrm{C} 2 "-\mathrm{C} 3 "$ & $1.542(5)$ & $\mathrm{C} 25^{\prime}-\mathrm{O} 93$ & $1.348(5)$ \\
\hline C3"-C4" & $1.492(7)$ & $\mathrm{C} 26^{\prime}-\mathrm{C} 27^{\prime}$ & $1.417(7)$ \\
\hline $\mathrm{C} 4 "-\mathrm{C} 4 " \mathrm{~A}$ & $1.498(7)$ & $\mathrm{C} 27^{\prime}-\mathrm{O} 94$ & $1.261(7)$ \\
\hline $\mathrm{C} 5 "-\mathrm{C} 4 " \mathrm{~A}$ & $1.324(8)$ & $\mathrm{C} 27^{\prime}-\mathrm{C} 28^{\prime}$ & $1.451(8)$ \\
\hline
\end{tabular}




$$
\begin{aligned}
& \mathrm{C} 5 "-\mathrm{O} 87 \\
& \mathrm{C} 5 "-\mathrm{C} 6 " \\
& \mathrm{C} 6 "-\mathrm{C} 7 " \\
& \mathrm{C} 7 "-\mathrm{C} 8 " \\
& \mathrm{C} 7 "-\mathrm{O} 86 \\
& \mathrm{C} 8 "-\mathrm{C} 8 " \mathrm{~A} \\
& \mathrm{C} 8 "-\mathrm{C} 3 \\
& \mathrm{C} 9 "-\mathrm{C} 10^{\prime \prime} \\
& \mathrm{C} 9 "-\mathrm{C} 14^{\prime \prime} \\
& \mathrm{C} 10^{\prime \prime}-\mathrm{C} 11^{\prime \prime}
\end{aligned}
$$

$$
\begin{array}{ll}
1.348(6) & \mathrm{C} 28^{\prime}-\mathrm{C} 29^{\prime} \\
1.434(7) & \mathrm{C} 29^{\prime}-\mathrm{O} 95 \\
1.376(7) & \mathrm{C} 30^{\prime}-\mathrm{C} 31^{\prime} \\
1.342(7) & \mathrm{C} 30^{\prime}-\mathrm{C} 35^{\prime} \\
1.363(6) & \mathrm{C} 31^{\prime}-\mathrm{C} 32^{\prime} \\
1.472(6) & \mathrm{C} 32^{\prime}-\mathrm{C} 33^{\prime} \\
1.515(5) & \mathrm{C} 33^{\prime}-\mathrm{C} 34^{\prime} \\
1.296(7) & \mathrm{C} 34^{\prime}-\mathrm{C} 35^{\prime} \\
1.397(7) & \mathrm{C} 4 \mathrm{~A}-\mathrm{C} 8 \mathrm{~A} \\
1.438(8) & \mathrm{C} 8 \mathrm{~A}-\mathrm{O} 1
\end{array}
$$

\begin{tabular}{|c|c|c|c|}
\hline $\mathrm{O} 1-\mathrm{C} 2-\mathrm{O} 80$ & $108.4(4)$ & $\mathrm{C} 29^{\prime \prime}-\mathrm{C} 34^{\prime \prime}-\mathrm{C} 33^{\prime \prime}$ & $118.9(5)$ \\
\hline $\mathrm{O} 1-\mathrm{C} 2-\mathrm{C} 9$ & $109.1(4)$ & $\mathrm{C} 5 "-\mathrm{C} 4 " \mathrm{~A}-\mathrm{C} 8 \mathrm{~A}$ & $120.9(5)$ \\
\hline $\mathrm{O} 80-\mathrm{C} 2-\mathrm{C} 9$ & $111.3(4)$ & $\mathrm{C} 5 "-\mathrm{C} 4 " \mathrm{~A}-\mathrm{C} 4 "$ & $122.0(5)$ \\
\hline $\mathrm{O} 1-\mathrm{C} 2-\mathrm{C} 3$ & $112.2(4)$ & $\mathrm{C} 8$ "A-C4"A-C4" & $117.1(4)$ \\
\hline $\mathrm{O} 80-\mathrm{C} 2-\mathrm{C} 3$ & $100.9(4)$ & $\mathrm{O} 1 "-\mathrm{C} 8 " \mathrm{~A}-\mathrm{C} 4 " \mathrm{~A}$ & $125.0(5)$ \\
\hline $\mathrm{C} 9-\mathrm{C} 2-\mathrm{C} 3$ & $114.7(4)$ & $\mathrm{O} 1 "-\mathrm{C} 8 " \mathrm{~A}-\mathrm{C} 8 "$ & $118.7(4)$ \\
\hline $\mathrm{C} 4-\mathrm{C} 3-\mathrm{C} 2$ & $113.1(4)$ & $\mathrm{C} 4 " \mathrm{~A}-\mathrm{C} 8 \mathrm{~A} \mathrm{~A}-\mathrm{C} 8 "$ & $116.3(4)$ \\
\hline $\mathrm{C} 4-\mathrm{C} 3-\mathrm{C}^{\prime}$ & $105.4(4)$ & $\mathrm{C} 8 " \mathrm{~B}-\mathrm{C} 4 " \mathrm{~B}-\mathrm{C} 25 "$ & $115.3(4)$ \\
\hline $\mathrm{C} 2-\mathrm{C} 3-\mathrm{C} 4{ }^{\prime}$ & $121.6(4)$ & C8"B-C4"B-C24" & $126.5(4)$ \\
\hline $\mathrm{C} 3-\mathrm{C} 4-\mathrm{C} 2{ }^{\prime}$ & $104.4(3)$ & $\mathrm{C} 25^{\prime \prime}-\mathrm{C} 4 " \mathrm{~B}-\mathrm{C} 24 "$ & $118.2(4)$ \\
\hline $\mathrm{C} 3-\mathrm{C} 4-\mathrm{C} 4 \mathrm{~A}$ & $111.8(3)$ & $\mathrm{C} 4 " \mathrm{~B}-\mathrm{C} 8 \mathrm{~B}-\mathrm{C} 28 "$ & $126.4(5)$ \\
\hline $\mathrm{C}^{\prime}-\mathrm{C} 4-\mathrm{C} 4 \mathrm{~A}$ & $111.5(3)$ & $\mathrm{C} 4 " \mathrm{~B}-\mathrm{C} 8 \mathrm{~B}-\mathrm{O} 21 "$ & $118.9(4)$ \\
\hline $\mathrm{O} 81-\mathrm{C} 5-\mathrm{C} 6$ & $119.4(5)$ & $\mathrm{C} 28 "-\mathrm{C} 8 " \mathrm{~B}-\mathrm{O} 21 "$ & $114.7(4)$ \\
\hline $\mathrm{O} 81-\mathrm{C} 5-\mathrm{C} 4 \mathrm{~A}$ & $119.4(4)$ & $\mathrm{O} 86-\mathrm{C} 1-\mathrm{O} 85$ & $107.4(3)$ \\
\hline $\mathrm{C} 6-\mathrm{C} 5-\mathrm{C} 4 \mathrm{~A}$ & $121.1(5)$ & $\mathrm{O} 86-\mathrm{C} 1{ }^{\prime}-\mathrm{C} 10^{\prime}$ & $108.7(4)$ \\
\hline $\mathrm{C} 5-\mathrm{C} 6-\mathrm{C} 7$ & $117.2(5)$ & $\mathrm{O} 85-\mathrm{C} 1{ }^{\prime}-\mathrm{C} 10^{\prime}$ & $107.6(3)$ \\
\hline $\mathrm{C} 5-\mathrm{C} 6-\mathrm{C} 81$ & $125.1(5)$ & $\mathrm{O} 86-\mathrm{C} 1^{\prime}-\mathrm{C} 2^{\prime}$ & $108.5(4)$ \\
\hline $\mathrm{C} 7-\mathrm{C} 6-\mathrm{C} 81$ & $117.7(4)$ & $\mathrm{O} 85-\mathrm{C} 1^{\prime}-\mathrm{C} 2{ }^{\prime}$ & $110.8(3)$ \\
\hline $\mathrm{C} 8-\mathrm{C} 7-\mathrm{C} 6$ & $122.5(5)$ & $\mathrm{C} 10^{\prime}-\mathrm{C} 1^{\prime}-\mathrm{C} 2^{\prime}$ & $113.6(4)$ \\
\hline $\mathrm{C} 8-\mathrm{C} 7-\mathrm{O} 82$ & $119.7(4)$ & $\mathrm{C} 3^{\prime}-\mathrm{C} 2^{\prime}-\mathrm{C} 4$ & $104.2(3)$ \\
\hline $\mathrm{C} 6-\mathrm{C} 7-\mathrm{O} 82$ & $117.7(5)$ & $\mathrm{C} 3^{\prime}-\mathrm{C} 2^{\prime}-\mathrm{C} 1^{\prime}$ & $108.2(4)$ \\
\hline $\mathrm{C} 8 \mathrm{~A}-\mathrm{C} 8-\mathrm{C} 7$ & $119.3(5)$ & $\mathrm{C} 4-\mathrm{C} 2{ }^{\prime}-\mathrm{C} 1^{\prime}$ & $112.6(4)$ \\
\hline $\mathrm{C} 14-\mathrm{C} 9-\mathrm{C} 10$ & $117.9(5)$ & $\mathrm{C} 2{ }^{\prime}-\mathrm{C} 3^{\prime}-\mathrm{C} 8^{\prime \prime}$ & $109.9(3)$ \\
\hline $\mathrm{C} 14-\mathrm{C} 9-\mathrm{C} 2$ & $121.0(5)$ & $\mathrm{C} 2{ }^{\prime}-\mathrm{C} 3^{\prime}-\mathrm{C} 4^{\prime}$ & $102.1(4)$ \\
\hline
\end{tabular}

1.315(7)

1.386(6)

1.387(6)

1.415(7)

1.417(7)

1.331(7)

1.431(7)

1.362(8)

1.375(7)

1.375(6)

(b) Bond Angles (deg): 


\begin{tabular}{|c|c|c|c|}
\hline $\mathrm{C} 10-\mathrm{C} 9-\mathrm{C} 2$ & $121.1(5)$ & $\mathrm{C} 8{ }^{\prime \prime}-\mathrm{C} 3^{\prime}-\mathrm{C} 4^{\prime}$ & $113.9(3)$ \\
\hline $\mathrm{C} 9-\mathrm{C} 10-\mathrm{C} 11$ & $123.9(5)$ & $\mathrm{C} 5^{\prime}-\mathrm{C} 4^{\prime}-\mathrm{C} 9^{\prime}$ & $115.1(5)$ \\
\hline $\mathrm{C} 10-\mathrm{C} 11-\mathrm{C} 12$ & $114.5(4)$ & $\mathrm{C} 5^{\prime}-\mathrm{C} 4^{\prime}-\mathrm{C} 3^{\prime}$ & $113.1(4)$ \\
\hline $\mathrm{C} 13-\mathrm{C} 12-\mathrm{C} 11$ & $120.0(5)$ & $\mathrm{C} 9^{\prime}-\mathrm{C} 4^{\prime}-\mathrm{C} 3^{\prime}$ & $107.6(4)$ \\
\hline $\mathrm{C} 12-\mathrm{C} 13-\mathrm{C} 14$ & $123.9(5)$ & $\mathrm{C} 5^{\prime}-\mathrm{C} 4^{\prime}-\mathrm{C} 3$ & $118.7(4)$ \\
\hline $\mathrm{C} 9-\mathrm{C} 14-\mathrm{C} 13$ & $119.8(5)$ & $\mathrm{C} 9^{\prime}-\mathrm{C} 4^{\prime}-\mathrm{C} 3$ & $100.4(3)$ \\
\hline $\mathrm{O} 21-\mathrm{C} 22-\mathrm{O} 90$ & $109.5(3)$ & $\mathrm{C} 3^{\prime}-\mathrm{C} 4^{\prime}-\mathrm{C} 3$ & $100.2(3)$ \\
\hline $\mathrm{O} 21-\mathrm{C} 22-\mathrm{C} 23$ & $113.2(4)$ & $\mathrm{O} 83-\mathrm{C} 5^{\prime}-\mathrm{C} 6^{\prime}$ & $124.0(5)$ \\
\hline $\mathrm{O} 90-\mathrm{C} 22-\mathrm{C} 23$ & $103.2(3)$ & $\mathrm{O} 83-\mathrm{C}^{\prime}-\mathrm{C}^{\prime}$ & $109.9(4)$ \\
\hline $\mathrm{O} 21-\mathrm{C} 22-\mathrm{C} 29$ & $106.2(3)$ & $\mathrm{C}^{\prime}-\mathrm{C} 5^{\prime}-\mathrm{C} 4^{\prime}$ & $126.1(5)$ \\
\hline $\mathrm{O} 90-\mathrm{C} 22-\mathrm{C} 29$ & $108.6(3)$ & $\mathrm{C} 5^{\prime}-\mathrm{C} 6{ }^{\prime}-\mathrm{C} 83$ & $131.8(5)$ \\
\hline $\mathrm{C} 23-\mathrm{C} 22-\mathrm{C} 29$ & $116.0(4)$ & $\mathrm{C}^{\prime}-\mathrm{C} 6^{\prime}-\mathrm{C} 7^{\prime}$ & $115.9(5)$ \\
\hline $\mathrm{C} 22-\mathrm{C} 23-\mathrm{C} 24^{\prime}$ & $123.1(3)$ & $\mathrm{C} 83-\mathrm{C}^{\prime}-\mathrm{C}^{\prime}$ & $112.0(4)$ \\
\hline $\mathrm{C} 22-\mathrm{C} 23-\mathrm{C} 24$ & $111.3(4)$ & $\mathrm{O} 84-\mathrm{C}^{\prime}-\mathrm{C} 8^{\prime}$ & $121.0(5)$ \\
\hline $\mathrm{C} 244^{\prime}-\mathrm{C} 23-\mathrm{C} 24$ & $104.5(3)$ & $\mathrm{O} 84-\mathrm{C}^{\prime}-\mathrm{C}^{\prime}$ & $119.8(5)$ \\
\hline $\mathrm{C} 43-\mathrm{C} 24-\mathrm{C} 22^{\prime}$ & $111.0(4)$ & $\mathrm{C} 8^{\prime}-\mathrm{C}^{\prime}-\mathrm{C} 6^{\prime}$ & $119.2(5)$ \\
\hline $\mathrm{C} 43-\mathrm{C} 24-\mathrm{C} 23$ & $113.0(4)$ & $\mathrm{C}^{\prime}-\mathrm{C} 8^{\prime}-\mathrm{C} 7^{\prime}$ & $120.8(4)$ \\
\hline $\mathrm{C} 22^{\prime}-\mathrm{C} 24-\mathrm{C} 23$ & $102.6(4)$ & $8^{\prime}-\mathrm{C} 9 '-\mathrm{O} 85$ & $119.5(4)$ \\
\hline $\mathrm{C} 43-\mathrm{C} 25-\mathrm{C} 26$ & $124.1(5)$ & $8^{\prime}-\mathrm{C} 9^{\prime}-\mathrm{C}^{\prime}$ & $122.7(5)$ \\
\hline $\mathrm{C} 43-\mathrm{C} 25-\mathrm{O} 91$ & $116.4(4)$ & $\mathrm{O} 85-\mathrm{C} 9^{\prime}-\mathrm{C} 4^{\prime}$ & $117.8(4)$ \\
\hline $\mathrm{C} 26-\mathrm{C} 25-\mathrm{O} 91$ & $119.4(5)$ & $\mathrm{C} 15^{\prime}-\mathrm{C} 10^{\prime}-\mathrm{C} 11^{\prime}$ & $119.7(5)$ \\
\hline $\mathrm{C} 27-\mathrm{C} 26-\mathrm{C} 25$ & $117.8(5)$ & $\mathrm{C} 15^{\prime}-\mathrm{C} 10^{\prime}-\mathrm{C} 1^{\prime}$ & $121.0(4)$ \\
\hline $\mathrm{C} 27-\mathrm{C} 26-\mathrm{C} 90$ & $123.4(5)$ & $\mathrm{C} 11^{\prime}-\mathrm{C} 10^{\prime}-\mathrm{C} 1^{\prime}$ & $119.3(4)$ \\
\hline $\mathrm{C} 25-\mathrm{C} 26-\mathrm{C} 90$ & $118.8(4)$ & $\mathrm{C} 12^{\prime}-\mathrm{C} 11^{\prime}-\mathrm{C} 10^{\prime}$ & $118.7(4)$ \\
\hline $\mathrm{O} 92-\mathrm{C} 27-\mathrm{C} 26$ & $117.3(5)$ & $\mathrm{C} 11^{\prime}-\mathrm{C} 12^{\prime}-\mathrm{C} 13^{\prime}$ & $121.5(5)$ \\
\hline $\mathrm{O} 92-\mathrm{C} 27-\mathrm{C} 28$ & $122.0(5)$ & $\mathrm{C} 14^{\prime}-\mathrm{C} 13^{\prime}-\mathrm{C} 12^{\prime}$ & $118.9(5)$ \\
\hline $\mathrm{C} 26-\mathrm{C} 27-\mathrm{C} 28$ & $120.7(5)$ & $\mathrm{C} 13^{\prime}-\mathrm{C} 14^{\prime}-\mathrm{C} 15^{\prime}$ & $119.4(6)$ \\
\hline $\mathrm{C} 27-\mathrm{C} 28-\mathrm{C} 48$ & $119.0(5)$ & $\mathrm{C} 10^{\prime}-\mathrm{C} 15^{\prime}-\mathrm{C} 14^{\prime}$ & $121.4(5)$ \\
\hline $\mathrm{C} 30-\mathrm{C} 29-\mathrm{C} 34$ & $118.8(5)$ & O96-C21'-O95 & $105.6(4)$ \\
\hline $\mathrm{C} 30-\mathrm{C} 29-\mathrm{C} 22$ & $123.3(4)$ & O96-C21'-C22' & $112.1(3)$ \\
\hline $\mathrm{C} 34-\mathrm{C} 29-\mathrm{C} 22$ & $117.8(4)$ & $\mathrm{O} 95-\mathrm{C} 21^{\prime}-\mathrm{C} 22^{\prime}$ & $112.9(3)$ \\
\hline $\mathrm{C} 29-\mathrm{C} 30-\mathrm{C} 31$ & $117.8(5)$ & O96-C21'-C30' & $104.3(4)$ \\
\hline $\mathrm{C} 32-\mathrm{C} 31-\mathrm{C} 30$ & $125.8(5)$ & $\mathrm{O} 95-\mathrm{C} 21^{\prime}-\mathrm{C} 30^{\prime}$ & $105.9(4)$ \\
\hline $\mathrm{C} 31-\mathrm{C} 32-\mathrm{C} 33$ & $117.3(5)$ & $\mathrm{C} 22^{\prime}-\mathrm{C} 21^{\prime}-\mathrm{C} 30^{\prime}$ & $115.2(3)$ \\
\hline C $34-\mathrm{C} 33-\mathrm{C} 32$ & $117.8(5)$ & $\mathrm{C} 21^{\prime}-\mathrm{C} 22^{\prime}-\mathrm{C} 24$ & $114.6(3)$ \\
\hline $\mathrm{C} 33-\mathrm{C} 34-\mathrm{C} 29$ & $122.5(5)$ & $\mathrm{C} 21^{\prime}-\mathrm{C} 22^{\prime}-\mathrm{C} 23^{\prime}$ & $107.6(3)$ \\
\hline $\mathrm{C} 48-\mathrm{C} 43-\mathrm{C} 25$ & $115.1(5)$ & $\mathrm{C} 24-\mathrm{C} 22^{\prime}-\mathrm{C} 23^{\prime}$ & $103.3(3)$ \\
\hline
\end{tabular}




\begin{tabular}{|c|c|c|c|}
\hline $\mathrm{C} 48-\mathrm{C} 43-\mathrm{C} 24$ & $121.8(5)$ & $\mathrm{C} 28^{\prime \prime}-\mathrm{C} 23^{\prime}-\mathrm{C} 24^{\prime}$ & $119.0(3)$ \\
\hline $\mathrm{C} 25-\mathrm{C} 43-\mathrm{C} 24$ & $123.1(5)$ & $\mathrm{C} 28^{\prime \prime}-\mathrm{C} 23^{\prime}-\mathrm{C} 22^{\prime}$ & $111.4(3)$ \\
\hline $\mathrm{O} 21-\mathrm{C} 48-\mathrm{C} 43$ & $123.2(4)$ & $\mathrm{C} 24^{\prime}-\mathrm{C} 23^{\prime}-\mathrm{C} 22^{\prime}$ & $96.5(3)$ \\
\hline $\mathrm{O} 21-\mathrm{C} 48-\mathrm{C} 28$ & $113.7(4)$ & $\mathrm{C} 29^{\prime}-\mathrm{C} 24^{\prime}-\mathrm{C} 23$ & $105.3(4)$ \\
\hline $\mathrm{C} 43-\mathrm{C} 48-\mathrm{C} 28$ & $123.1(4)$ & $\mathrm{C} 29^{\prime}-\mathrm{C} 24^{\prime}-\mathrm{C} 23^{\prime}$ & $105.7(4)$ \\
\hline $\mathrm{O} 1 "-\mathrm{C} 2 "-\mathrm{C} 9 "$ & $111.2(3)$ & $\mathrm{C} 23-\mathrm{C} 24^{\prime}-\mathrm{C} 23^{\prime}$ & $108.2(3)$ \\
\hline $\mathrm{O} 1 "-\mathrm{C} 2 "-\mathrm{C} 3 "$ & $107.9(3)$ & $\mathrm{C} 29^{\prime}-\mathrm{C} 24^{\prime}-\mathrm{C} 25^{\prime}$ & $108.9(4)$ \\
\hline $\mathrm{C} 9 "-\mathrm{C} 2 "-\mathrm{C} 3 "$ & $112.0(3)$ & $\mathrm{C} 23-\mathrm{C} 24^{\prime}-\mathrm{C} 25^{\prime}$ & $119.5(4)$ \\
\hline $\mathrm{C} 4 "-\mathrm{C} 3 "-\mathrm{C} 2 "$ & $110.5(4)$ & $\mathrm{C} 23^{\prime}-\mathrm{C} 24^{\prime}-\mathrm{C} 25^{\prime}$ & $108.5(4)$ \\
\hline $\mathrm{C} 3 "-\mathrm{C} 4 "-\mathrm{C} 4 " \mathrm{~A}$ & $112.1(4)$ & $\mathrm{C} 26^{\prime}-\mathrm{C} 25^{\prime}-\mathrm{O} 93$ & $131.5(4)$ \\
\hline $\mathrm{C} 4 " \mathrm{~A}-\mathrm{C} 5 "-\mathrm{O} 87$ & $116.0(5)$ & $\mathrm{C} 26^{\prime}-\mathrm{C} 25^{\prime}-\mathrm{C} 24^{\prime}$ & $123.7(4)$ \\
\hline $\mathrm{C} 4 " \mathrm{~A}-\mathrm{C} 5 "-\mathrm{C} 6 "$ & $122.8(5)$ & $\mathrm{O} 93-\mathrm{C} 25^{\prime}-\mathrm{C} 24^{\prime}$ & $104.6(3)$ \\
\hline O87-C5"-C6" & $121.1(4)$ & $\mathrm{C} 25^{\prime}-\mathrm{C} 26^{\prime}-\mathrm{C} 27^{\prime}$ & $120.1(5)$ \\
\hline C7"-C6"- 5 " & $116.2(4)$ & $\mathrm{C} 25^{\prime}-\mathrm{C} 26^{\prime}-\mathrm{C} 93$ & $124.0(5)$ \\
\hline $\mathrm{C} 8 "-\mathrm{C} 7 "-\mathrm{O} 86$ & $121.6(4)$ & $\mathrm{C} 27^{\prime}-\mathrm{C} 26^{\prime}-\mathrm{C} 93$ & $115.9(5)$ \\
\hline C8"-C7"-C6" & $125.0(5)$ & O94-C27'-C26' & $121.1(5)$ \\
\hline O86-C7"- 6 - & $113.4(4)$ & O94-C27'-C28' & $119.6(4)$ \\
\hline $\mathrm{C} 7 "-\mathrm{C} 8 "-\mathrm{C} 8 " \mathrm{~A}$ & $118.7(4)$ & $\mathrm{C} 26^{\prime}-\mathrm{C} 27^{\prime}-\mathrm{C} 28^{\prime}$ & $119.3(5)$ \\
\hline $\mathrm{C} 7 "-\mathrm{C} 8{ }^{\prime \prime}-\mathrm{C} 3^{\prime}$ & $121.0(4)$ & $\mathrm{C} 29^{\prime}-\mathrm{C} 28^{\prime}-\mathrm{C} 27^{\prime}$ & $120.5(4)$ \\
\hline $\mathrm{C} 8 " \mathrm{~A}-\mathrm{C} 8 "-\mathrm{C} 3^{\prime}$ & $120.2(3)$ & $\mathrm{C} 28^{\prime}-\mathrm{C} 29^{\prime}-\mathrm{O} 95$ & $120.0(4)$ \\
\hline C10"-C9"-C14" & $115.6(4)$ & $\mathrm{C} 28^{\prime}-\mathrm{C} 29^{\prime}-\mathrm{C} 24^{\prime}$ & $125.1(5)$ \\
\hline $\mathrm{C} 10 "-\mathrm{C} 9 "-\mathrm{C} 2 "$ & $125.7(5)$ & O95-C29'-C24' & $113.8(4)$ \\
\hline $\mathrm{C} 14 "-\mathrm{C} 9 "-\mathrm{C} 2 "$ & $118.2(4)$ & $\mathrm{C} 31^{\prime}-\mathrm{C} 30^{\prime}-\mathrm{C} 35^{\prime}$ & $118.7(5)$ \\
\hline C9"-C10"-C11" & $125.5(5)$ & $\mathrm{C} 31^{\prime}-\mathrm{C} 30^{\prime}-\mathrm{C} 21^{\prime}$ & $120.7(4)$ \\
\hline $\mathrm{C} 12 "-\mathrm{C} 11 "-\mathrm{C} 10 "$ & $113.1(5)$ & $\mathrm{C} 35^{\prime}-\mathrm{C} 30^{\prime}-\mathrm{C} 21^{\prime}$ & $120.6(4)$ \\
\hline $\mathrm{C} 11 "-\mathrm{C} 12 "-\mathrm{C} 13 "$ & $124.3(5)$ & $\mathrm{C} 30^{\prime}-\mathrm{C} 31^{\prime}-\mathrm{C} 32^{\prime}$ & $120.7(5)$ \\
\hline $\mathrm{C} 14 "-\mathrm{C} 13^{\prime \prime}-\mathrm{C} 12^{\prime \prime}$ & $117.2(5)$ & $\mathrm{C} 33^{\prime}-\mathrm{C} 32^{\prime}-\mathrm{C} 31^{\prime}$ & $120.5(5)$ \\
\hline C13"-C14"-C9" & $124.0(5)$ & $\mathrm{C} 32^{\prime}-\mathrm{C} 33^{\prime}-\mathrm{C} 34^{\prime}$ & $119.3(5)$ \\
\hline $\mathrm{O} 21^{\prime \prime}-\mathrm{C} 22^{\prime \prime}-\mathrm{C} 23^{\prime \prime}$ & $110.6(0)$ & $\mathrm{C} 35^{\prime}-\mathrm{C} 34^{\prime}-\mathrm{C} 33^{\prime}$ & $121.3(5)$ \\
\hline $\mathrm{O} 21 "-\mathrm{C} 22 "-\mathrm{C} 29 "$ & $106.2(3)$ & $\mathrm{C} 34^{\prime}-\mathrm{C} 35^{\prime}-\mathrm{C} 30^{\prime}$ & $119.4(5)$ \\
\hline C23"-C22"-C29" & $115.8(3)$ & $\mathrm{C} 8 \mathrm{~A}-\mathrm{C} 4 \mathrm{~A}-\mathrm{C} 5$ & $118.7(5)$ \\
\hline C22"-C23"-C24" & $110.7(4)$ & $\mathrm{C} 8 \mathrm{~A}-\mathrm{C} 4 \mathrm{~A}-\mathrm{C} 4$ & $121.6(5)$ \\
\hline $\mathrm{C} 4 " \mathrm{~B}-\mathrm{C} 24 "-\mathrm{C} 23 "$ & $108.4(4)$ & $\mathrm{C} 5-\mathrm{C} 4 \mathrm{~A}-\mathrm{C} 4$ & $119.7(4)$ \\
\hline C26"-C25"-O97 & $126.2(4)$ & $\mathrm{C} 8-\mathrm{C} 8 \mathrm{~A}-\mathrm{C} 4 \mathrm{~A}$ & $121.0(5)$ \\
\hline C26"-C25"-C4"B & $121.2(5)$ & $\mathrm{C} 8-\mathrm{C} 8 \mathrm{~A}-\mathrm{O} 1$ & $116.1(4)$ \\
\hline O97-C25"-C4"B & $112.6(4)$ & $\mathrm{C} 4 \mathrm{~A}-\mathrm{C} 8 \mathrm{~A}-\mathrm{O} 1$ & $122.9(4)$ \\
\hline $\mathrm{C} 25 "-\mathrm{C} 26 "-\mathrm{C} 27 "$ & $118.9(5)$ & $\mathrm{C} 8 \mathrm{~A}-\mathrm{O} 1-\mathrm{C} 2$ & $116.3(4)$ \\
\hline
\end{tabular}




\begin{tabular}{|c|c|c|c|}
\hline $\mathrm{C} 26 "-\mathrm{C} 27 "-\mathrm{O} 96$ & $112.7(4)$ & $\mathrm{C} 48-\mathrm{O} 21-\mathrm{C} 22$ & $115.7(3)$ \\
\hline $\mathrm{C} 26 "-\mathrm{C} 27 "-\mathrm{C} 28 "$ & $122.9(4)$ & $\mathrm{C} 5-\mathrm{O} 81-\mathrm{C} 80$ & $116.0(4)$ \\
\hline O96-C27"-C28" & $124.3(4)$ & $\mathrm{C} 5-\mathrm{O} 83-\mathrm{C} 82$ & $124.2(4)$ \\
\hline $\mathrm{C} 8 \mathrm{~B}-\mathrm{C} 28 "-\mathrm{C} 27 "$ & $115.2(4)$ & $\mathrm{C} 9^{\prime}-\mathrm{O} 85-\mathrm{C} 1^{\prime}$ & $119.4(3)$ \\
\hline $\mathrm{C} 8{ }^{\prime \prime} \mathrm{B}-\mathrm{C} 28^{\prime \prime}-\mathrm{C} 23^{\prime}$ & $127.0(4)$ & $\mathrm{C} 7 "-\mathrm{O} 86-\mathrm{C}^{\prime}$ & $120.7(4)$ \\
\hline $\mathrm{C} 27^{\prime \prime}-\mathrm{C} 28^{\prime \prime}-\mathrm{C} 23^{\prime}$ & $117.8(4)$ & $\mathrm{C} 5 "-\mathrm{O} 87-\mathrm{C} 84$ & $122.1(4)$ \\
\hline C34"-C29"-C30" & $118.7(4)$ & $\mathrm{C} 91-\mathrm{O} 91-\mathrm{C} 25$ & $113.5(3)$ \\
\hline C34"-C29"-C22" & $119.5(0)$ & $\mathrm{C} 25^{\prime}-\mathrm{O} 93-\mathrm{C} 92$ & $122.1(3)$ \\
\hline C30"-C29"-C22" & $121.5(4)$ & $\mathrm{C} 29^{\prime}-\mathrm{O} 95-\mathrm{C} 21^{\prime}$ & $117.3(3)$ \\
\hline C31"-C30"-C29" & $117.9(5)$ & $\mathrm{C} 27^{\prime \prime}-\mathrm{O} 96-\mathrm{C} 21^{\prime}$ & $116.4(3)$ \\
\hline C30"-C31"-C32" & $127.6(5)$ & C25"-O97-C94 & $113.8(4)$ \\
\hline C33"-C32"-C31" & $113.7(5)$ & $\mathrm{C} 8 " \mathrm{~A}-\mathrm{O} 1 "-\mathrm{C} 2 "$ & $119.4(4)$ \\
\hline C32"-C33"-C34" & $123.0(5)$ & $\mathrm{C} 8 \mathrm{~B} \mathrm{~B}-\mathrm{O} 21 "-\mathrm{C} 22 "$ & $113.1(3)$ \\
\hline
\end{tabular}

Fig. S1. An ORTEP diagram of a merged dragonbloodin A $(\mathbf{1}+\mathbf{2})$ molecule is presented with thermal ellipsoids for non-hydrogen atoms shown at $15 \%$ probability level. Carbon, oxygen, and hydrogen atoms are shown as gray, red, and white spheres, respectively. All hydrogen atoms except those connected to oxygen atoms are omitted for clarity. The carbon label, C8a, for the atom connected to atoms C8, O1, and C4a, is not shown. One carbon atom was found disordered in two positions with $\mathrm{C} 2$ " $\mathrm{X}$ and C2"Y at occupancies of 0.616 and 0.384 , respectively.

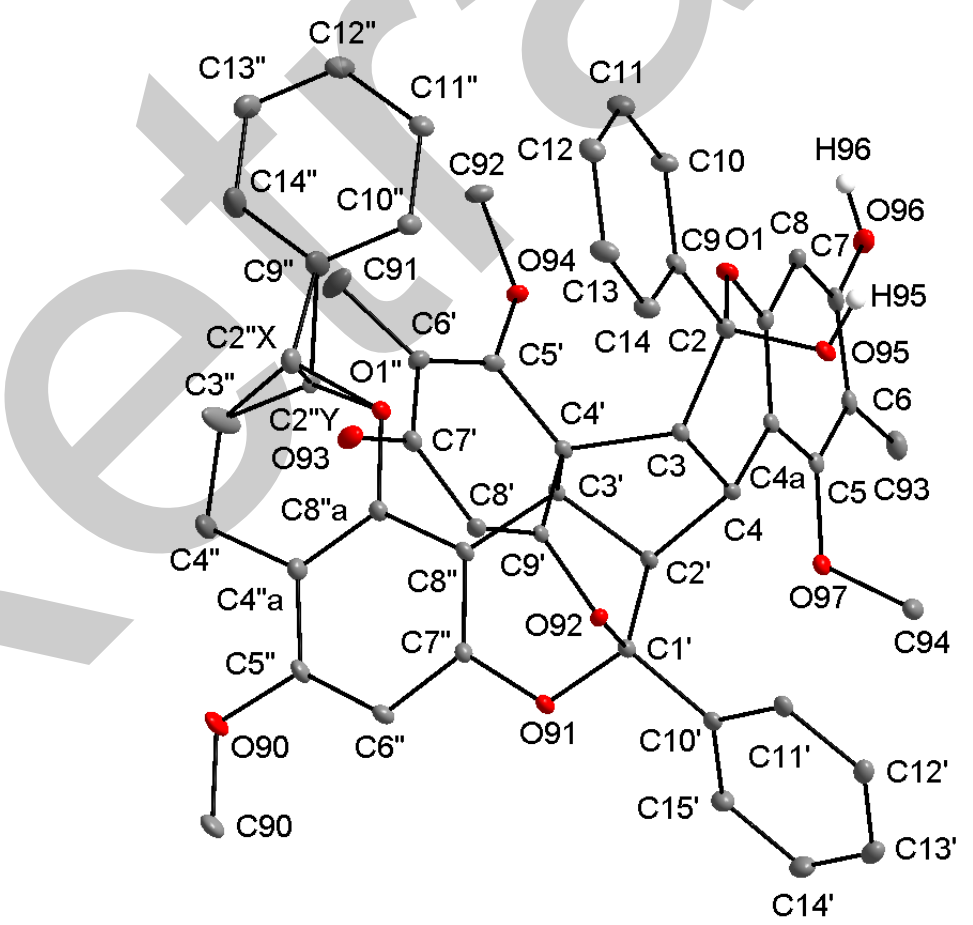


Spectroscopic data of 1 and 2

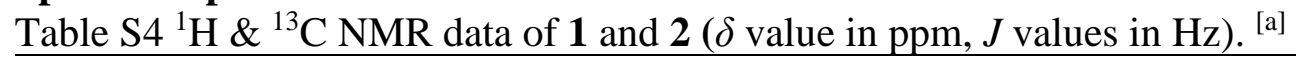

\begin{tabular}{|c|c|c|c|c|}
\hline \multirow[t]{2}{*}{ No. } & \multicolumn{2}{|l|}{1} & \multicolumn{2}{|l|}{2} \\
\hline & $\delta_{\mathrm{H}}$ & $\delta_{\mathrm{C}}$ & $\delta_{\mathrm{H}}$ & $\delta_{\mathrm{C}}$ \\
\hline 2 & - & $96.91(\mathrm{~s})$ & - & $96.86(\mathrm{~s})$ \\
\hline 3 & $3.90(\mathrm{~d}, 9.1)$ & $51.85(\mathrm{~d})$ & $3.86(\mathrm{~d}, 9.1)$ & $52.52(\mathrm{~d})$ \\
\hline 4 & $3.75(\mathrm{~d}, 9.1)$ & $35.81(\mathrm{~d})$ & $3.74(\mathrm{~d}, 9.1)$ & 35.49 (d) \\
\hline $4 a$ & - & $109.59(\mathrm{~s})$ & - & $109.49(\mathrm{~s})$ \\
\hline 5 & - & $157.37(\mathrm{~s})$ & - & $157.51(\mathrm{~s})$ \\
\hline 6 & - & $111.36(\mathrm{~s})$ & - & $111.38(\mathrm{~s})$ \\
\hline 7 & - & $156.04(\mathrm{~s})$ & - & $156.03(\mathrm{~s})$ \\
\hline 8 & $6.40(\mathrm{~s})$ & $100.37(\mathrm{~d})$ & $6.40(\mathrm{~s})$ & 100.57 (d) \\
\hline $8 a$ & - & $152.40(\mathrm{~d})$ & - & $152.31(\mathrm{~d})$ \\
\hline 9 & - & $143.04(\mathrm{~s})$ & - & $141.45(\mathrm{~s})$ \\
\hline 10 & $7.25-7.30(\mathrm{~m})$ & $128.76(\mathrm{~d})$ & $7.14-7.18(\mathrm{~m})$ & $128.80(d)$ \\
\hline 11 & $7.25-7.30(\mathrm{~m})$ & $128.76(\mathrm{~d})$ & $7.14-7.18(\mathrm{~m})$ & $128.80(d)$ \\
\hline 12 & $7.25-7.30(\mathrm{~m})$ & $128.76(\mathrm{~d})$ & $7.14-7.18(\mathrm{~m})$ & $128.80(\mathrm{~d})$ \\
\hline 13 & $7.25-7.30(\mathrm{~m})$ & $128.76(\mathrm{~d})$ & $7.14-7.18(\mathrm{~m})$ & $128.80(d)$ \\
\hline 14 & $7.25-7.30(\mathrm{~m})$ & $128.76(\mathrm{~d})$ & $7.14-7.18(\mathrm{~m})$ & $128.80(d)$ \\
\hline 2-OH & $5.41(\mathrm{~s})$ & - & $5.41(\mathrm{~s})$ & \\
\hline 5-OMe & $2.93(\mathrm{~s})$ & $59.08(\mathrm{q})$ & $2.92(\mathrm{~s})$ & $59.08(q)$ \\
\hline 6-Me & $2.01(\mathrm{~s})$ & $8.42(\mathrm{q})$ & $1.98(\mathrm{~s})$ & $8.38(q)$ \\
\hline 7-OH & $8.33(\mathrm{~s})$ & - & $8.28(\mathrm{~s}, \mathrm{br})$ & \\
\hline $1^{\prime}$ & - & $104.58(\mathrm{~s})$ & - & $104.52(\mathrm{~s})$ \\
\hline $2^{\prime}$ & $3.21(\mathrm{~d}, 4.6)$ & $49.20(\mathrm{~d})$ & $3.21(\mathrm{~d}, 4.9)$ & 49.56 (d) \\
\hline $3^{\prime}$ & $4.03(\mathrm{~d}, 4.6)$ & 38.49 (d) & $3.90(\mathrm{~d}, 4.9)$ & 38.40 (d) \\
\hline $4^{\prime}$ & - & $54.68(\mathrm{~s})$ & & $54.42(\mathrm{~s})$ \\
\hline $5^{\prime}$ & - & $164.17(\mathrm{~s})$ & & $163.43(\mathrm{~s})$ \\
\hline $6^{\prime}$ & - & $112.55(\mathrm{~s})$ & & $113.02(\mathrm{~s})$ \\
\hline $7^{\prime}$ & - & $187.71(\mathrm{~s})$ & & $187.43(\mathrm{~s})$ \\
\hline $8^{\prime}$ & $5.42(\mathrm{~s})$ & $106.30(d)$ & $5.42(\mathrm{~s})$ & $105.60(d)$ \\
\hline $9^{\prime}$ & - & $171.74(\mathrm{~s})$ & - & $171.89(\mathrm{~s})$ \\
\hline $10^{\prime}$ & - & $139.72(\mathrm{~s})$ & - & $139.75(\mathrm{~s})$ \\
\hline $11^{\prime}$ & $7.89(\mathrm{~d}, 7.4)$ & $127.15(\mathrm{~d})$ & $7.90(\mathrm{~d}, 7.4)$ & $127.20(\mathrm{~d})$ \\
\hline $12^{\prime}$ & $7.63(\mathrm{t}, 7.7)$ & $128.80(\mathrm{~d})$ & $7.64(t, 7.7)$ & $128.80(d)$ \\
\hline $13^{\prime}$ & $7.55(\mathrm{t}, 7.7)$ & 129.49 (d) & $7.55(\mathrm{t}, 7.7)$ & $129.52(d)$ \\
\hline $14^{\prime}$ & $7.63(t, 7.7)$ & $128.80(\mathrm{~d})$ & $7.64(t, 7.7)$ & $128.80(d)$ \\
\hline $15^{\prime}$ & $7.89(\mathrm{~d}, 7.4)$ & $127.15(\mathrm{~d})$ & $7.90(\mathrm{~d}, 7.6)$ & $127.20(d)$ \\
\hline 5'-OMe & $2.89(\mathrm{~s})$ & $59.16(q)$ & $3.20(\mathrm{~s})$ & $58.50(\mathrm{q})$ \\
\hline $6^{\prime}-\mathrm{Me}$ & $1.17(\mathrm{~s})$ & $9.24(\mathrm{q})$ & $0.70(\mathrm{~s})$ & $9.24(q)$ \\
\hline $2^{\prime \prime}$ & $5.02(\mathrm{dd}, 9.2,2.6)$ & $77.64(d)$ & $5.31(\mathrm{dd}, 5.2,4.0)$ & $77.02(d)$ \\
\hline 3" & $2.01-2.05(\mathrm{~m})$ & $29.97(t)$ & $1.98-2.05(\mathrm{~m})$ & $26.56(t)$ \\
\hline & $1.71(\mathrm{~m})$ & & $1.98-2.05(\mathrm{~m})$ & \\
\hline $4^{\prime \prime}$ & $2.53(\mathrm{ddd}, 16.0,5.7,4.6)$ & $18.85(\mathrm{t})$ & $2.50(\mathrm{ddd}, 16.8,6.2,4.9)$ & $15.92(t)$ \\
\hline & $2.45(\mathrm{ddd}, 17.2,10.5,5.9)$ & & $1.94-1.98(\mathrm{~m})$ & \\
\hline $4^{\prime \prime a}$ & - & $104.22(\mathrm{~s})$ & - & $104.64(\mathrm{~s})$ \\
\hline $5^{\prime \prime}$ & - & $158.11(\mathrm{~s})$ & - & $158.15(\mathrm{~s})$ \\
\hline $6^{\prime \prime}$ & $6.07(\mathrm{~s})$ & 91.56 (d) & $6.10(\mathrm{~s})$ & $91.50(\mathrm{~d})$ \\
\hline $7^{\prime \prime}$ & - & $152.83(\mathrm{~s})$ & - & $152.97(\mathrm{~s})$ \\
\hline $8^{\prime \prime}$ & - & $100.97(\mathrm{~s})$ & - & $100.80(\mathrm{~s})$ \\
\hline $8^{\prime \prime} \mathrm{a}$ & - & $153.77(\mathrm{~s})$ & - & $154.07(\mathrm{~s})$ \\
\hline 9" & - & $141.84(\mathrm{~s})$ & - & $142.17(\mathrm{~s})$ \\
\hline $10^{\prime \prime}$ & $7.20-7.23(\mathrm{~m})$ & $126.03(\mathrm{~d})$ & $7.05(\mathrm{~d}, 7.1)$ & 126.29 (d) \\
\hline $11^{\prime \prime}$ & $7.20-7.23(\mathrm{~m})$ & $127.70(\mathrm{~d})$ & $7.19-7.24(\mathrm{~m})$ & $127.50(d)$ \\
\hline $12^{\prime \prime}$ & 7.24-7.25 (m) & $128.36(\mathrm{~d})$ & $7.19-7.24(\mathrm{~m})$ & $128.25(\mathrm{~d})$ \\
\hline $13^{\prime \prime}$ & 7.20-7.23 (d,7.6) & $127.70(\mathrm{~d})$ & $7.19-7.24(\mathrm{~m})$ & $127.50(\mathrm{~d})$ \\
\hline
\end{tabular}




\begin{tabular}{llrlr}
\hline $\mathbf{1 4}^{\prime \prime}$ & $7.20-7.23(\mathrm{~m})$ & $126.03(\mathrm{~d})$ & $7.05(\mathrm{~d}, 7.1)$ & $126.29(\mathrm{~d})$ \\
$\mathbf{5}^{\prime \prime}$-OMe & $3.71(\mathrm{~s})$ & $55.38(\mathrm{q})$ & $3.71(\mathrm{~s})$ & $55.36(\mathrm{q})$ \\
\hline
\end{tabular}

[a] ${ }^{1} \mathrm{H}$ NMR and ${ }^{13} \mathrm{C}$ NMR in $\mathrm{d}_{6}$-Acetone.

\section{Physico-chemical constants of Dragonbloodin A1 (1) and A2 (2)}

Dragonbloodin A1 (1). Colorless amorphous powder. Optical rotation $\left([\alpha]^{26} \mathrm{D}\right)+42.3619$ (c 0.09, $\mathrm{CHCl}_{3}$ ); UV (log $\varepsilon$, in $\left.\mathrm{CHCl}_{3}\right) \lambda_{\max } 282.0$ (3.84), 247.0 (4.20) nm; IR (KBr) $v_{\max }$ 3372, 2925, 2854, 1667, 1615, 1600, 1448, 1367, 1120, $699 \mathrm{~cm}^{-1}$; ECD (Mol. CD, in $\left.\mathrm{CHCl}_{3}\right) 306(+11.1917), 271$ (+2.16617), 249 (-6.10281), $221(-1.84619), 211$ $(+0.96840) \mathrm{nm}$; HR-ESI-MS (m/z 805.3003 [M+H] $]^{+}$, calcd for $\left.\mathrm{C}_{50} \mathrm{H}_{45} \mathrm{O}_{10}: 805.3007\right)$.

Dragonbloodin A2 (2). Colorless amorphous powder. Optical rotation $\left([\alpha]^{26} \mathrm{D}\right)-58.0865$ (c 0.06, $\mathrm{CHCl}_{3}$ ); UV (loge, in $\mathrm{CHCl}_{3}$ ) $\lambda_{\max } 315.0$ (3.26), 283.0 (3.76), 247.0 (4.18) nm; IR $(\mathrm{KBr}) v_{\max } 3374,2925,2854,1667,1615,1601,1448,1368,1121,700 \mathrm{~cm}^{-1}$; ECD (Mol. CD, in $\left.\mathrm{CHCl}_{3}\right) 306$ (-7.79742), 270 (-3.08468), 248 (+5.38757), 222 (-1.24149), $211(+1.25683) \mathrm{nm}$; HR-ESI-MS $\left(\mathrm{m} / \mathrm{z} 805.3007[\mathrm{M}+\mathrm{H}]^{+}\right.$, calcd for $\mathrm{C}_{50} \mathrm{H}_{45} \mathrm{O}_{10}$ : 805.3007).

Fig. S2. Key ${ }^{1} \mathrm{H}_{-}{ }^{1} \mathrm{H}$ COSY correlations of Dragonbloodin A1 (1)

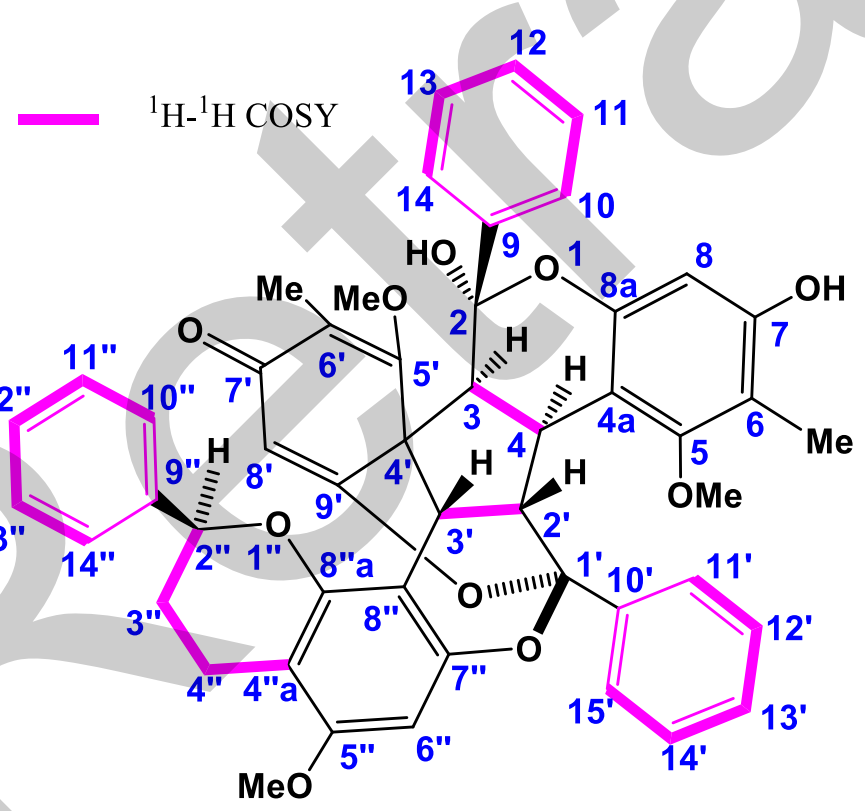


Fig. S3. Key HMBC correlations of Dragonbloodin A1 (1)
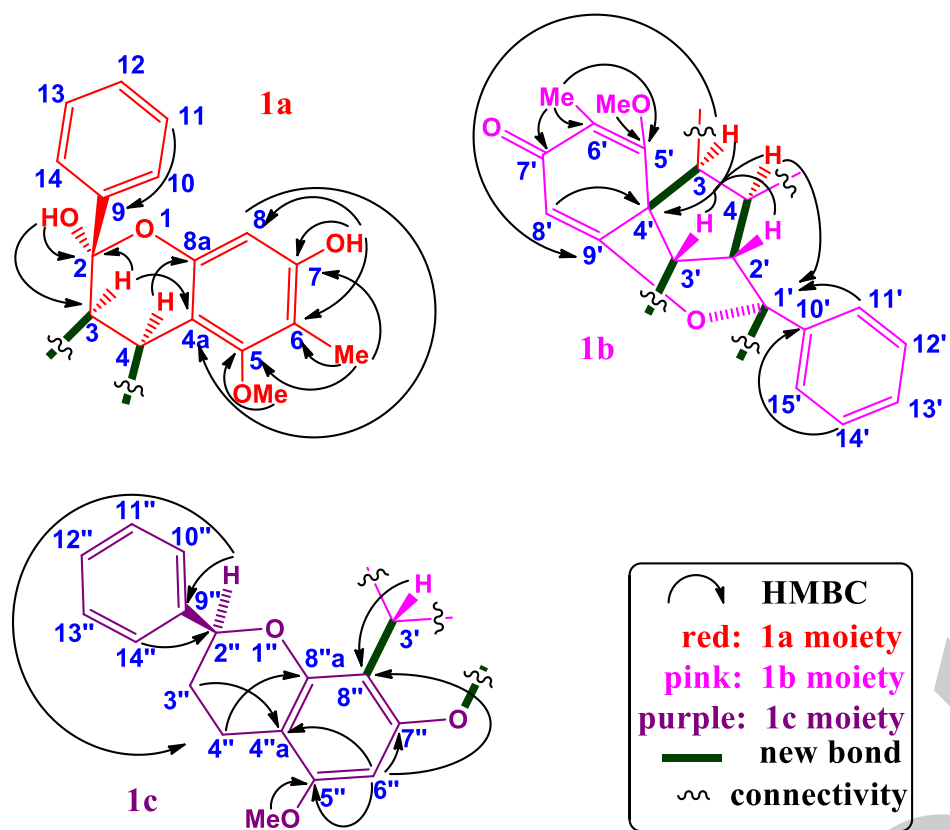

\section{Miscellaneous spectra}

Fig. S4. The ECD spectrum of $\mathbf{1}$ and $\mathbf{2}$ had opposite peaks to each other.

Dragonbloodin $\mathrm{A} 1$ (in $\mathrm{CHCl}_{3}$ )

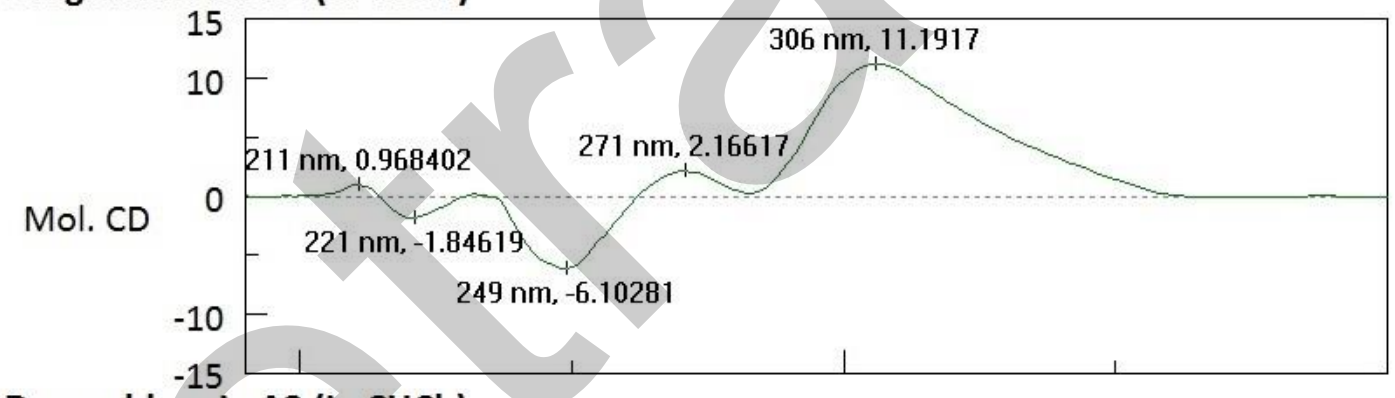

Dragonbloonin A2 (in $\mathrm{CHCl}_{3}$ )

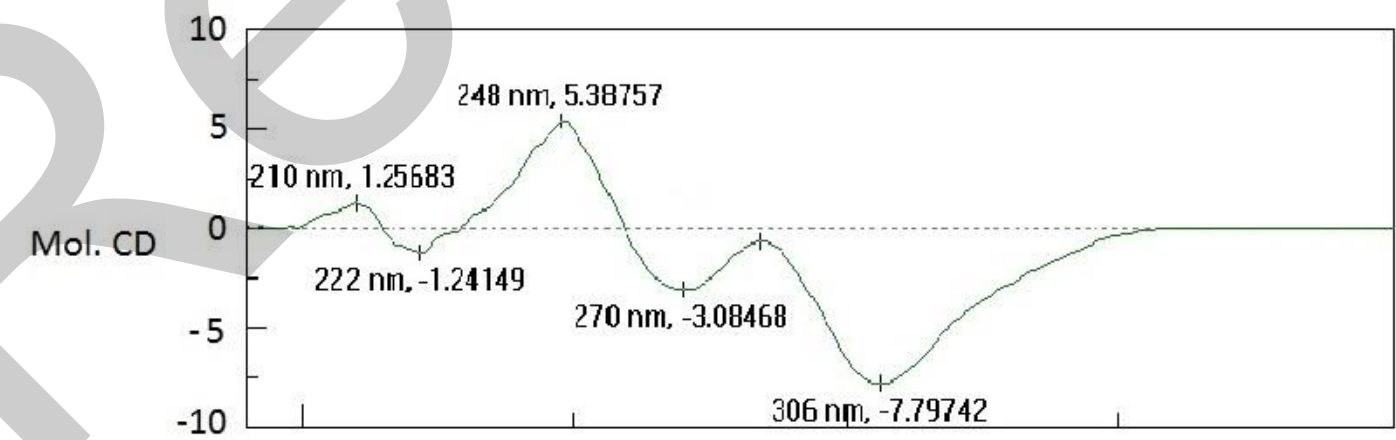


Fig. S5. UV spectrum of 1 in $\mathrm{CHCl} 3$

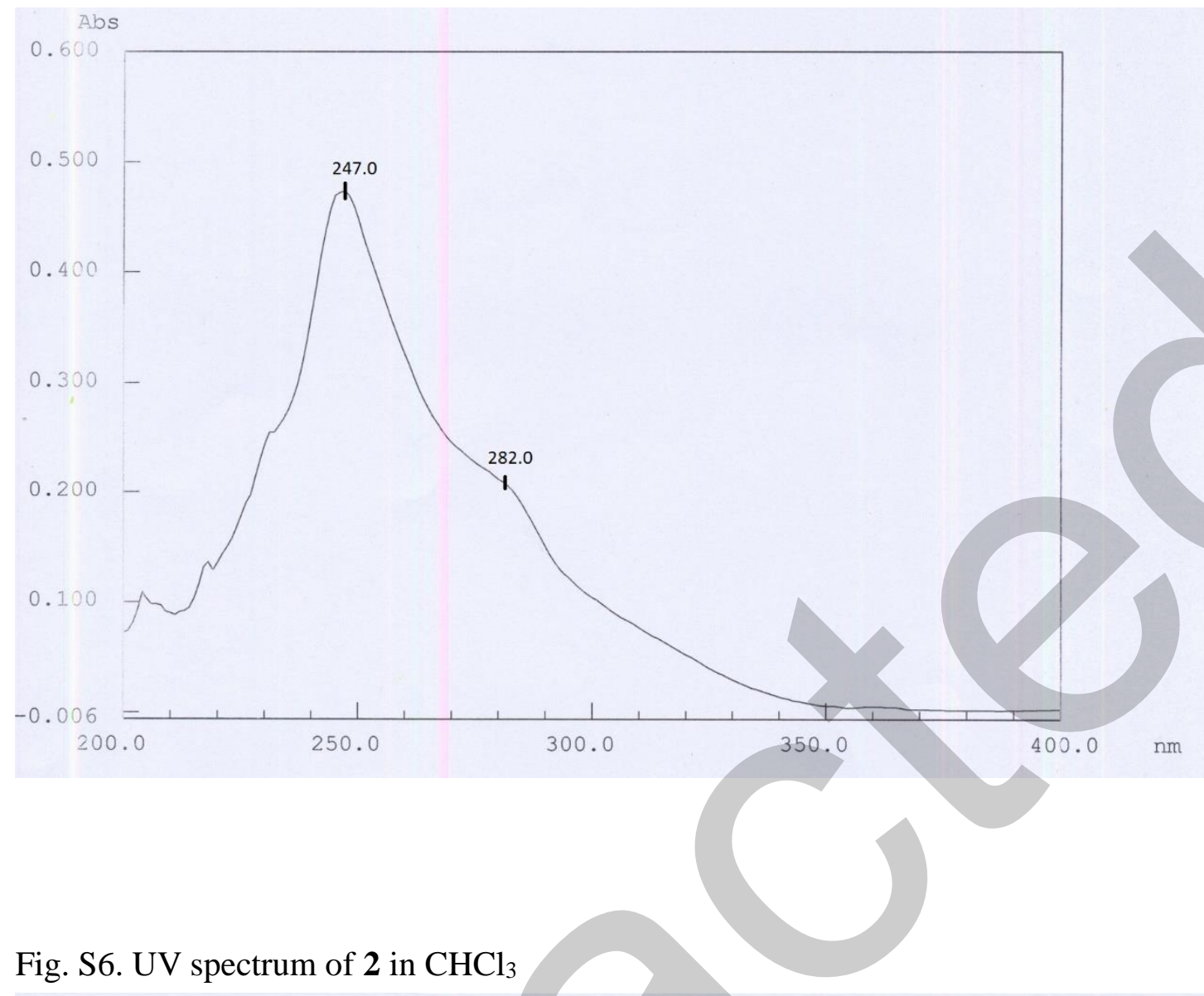

Fig. S6. UV spectrum of 2 in $\mathrm{CHCl}_{3}$

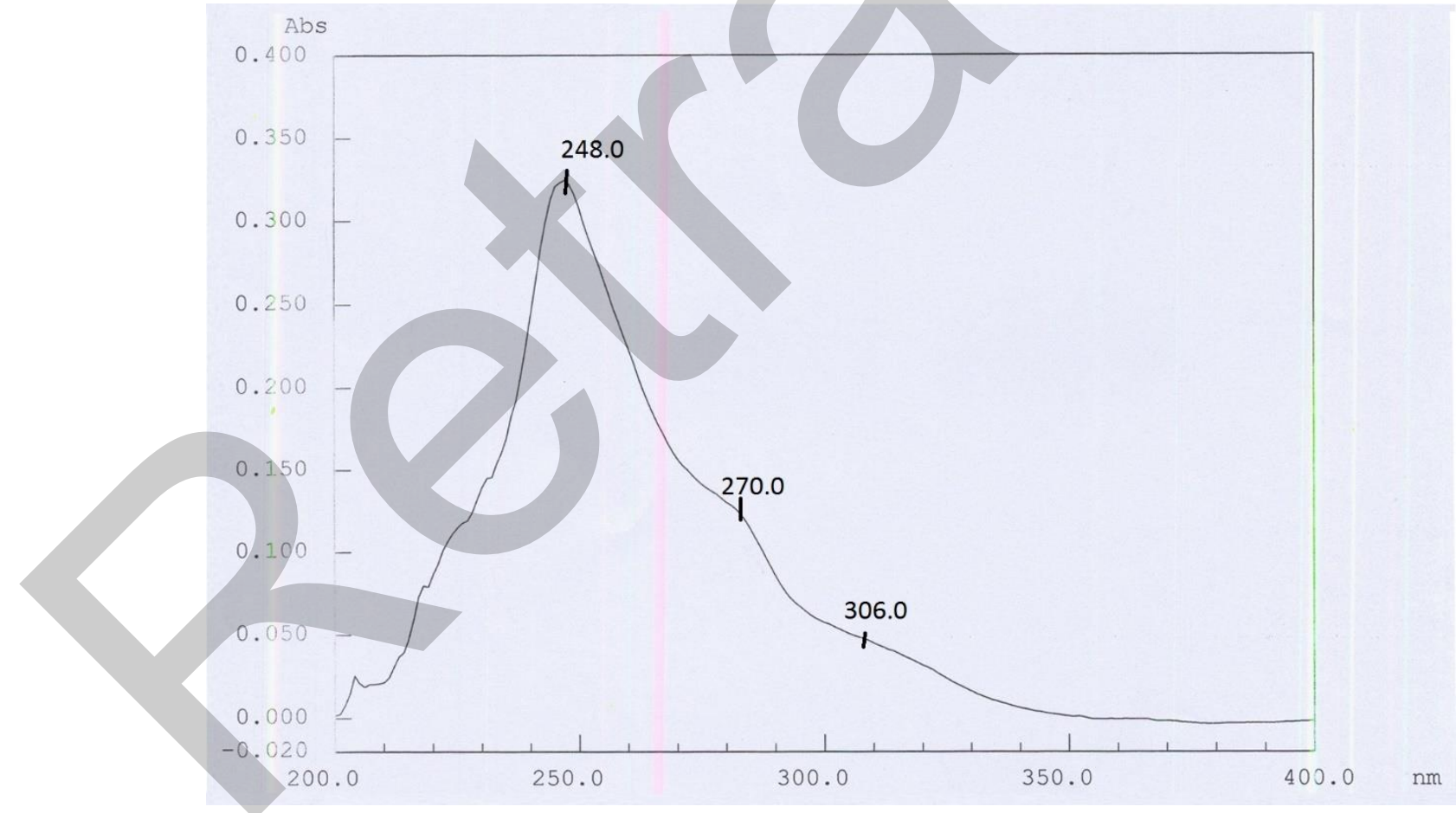




$$
e^{e^{e^{e^{e}}}}
$$


Fig. S9. HR-ESI-Mass of $\mathbf{1}$

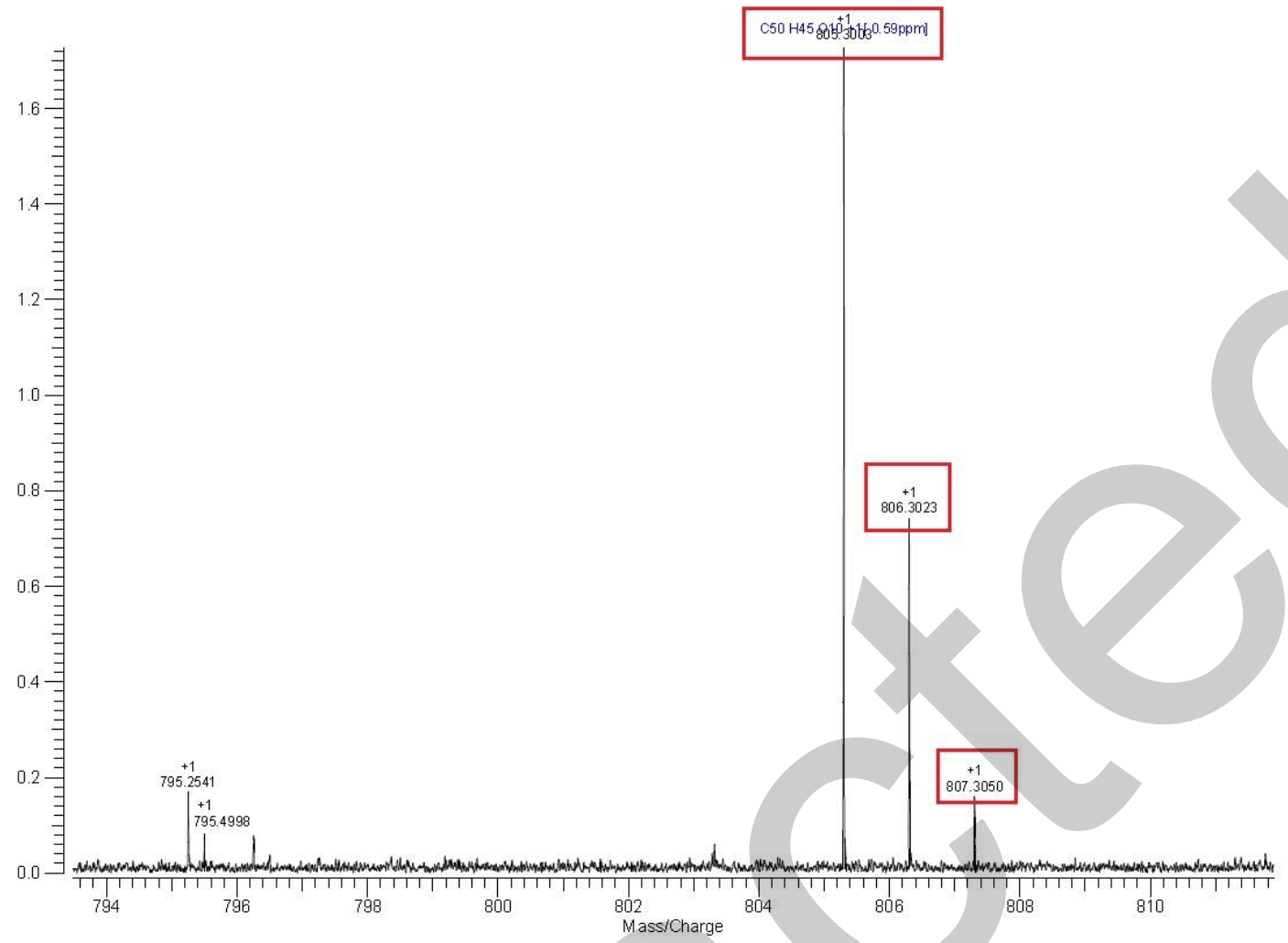

Fig. S10. HR-ESI-Mass of 2

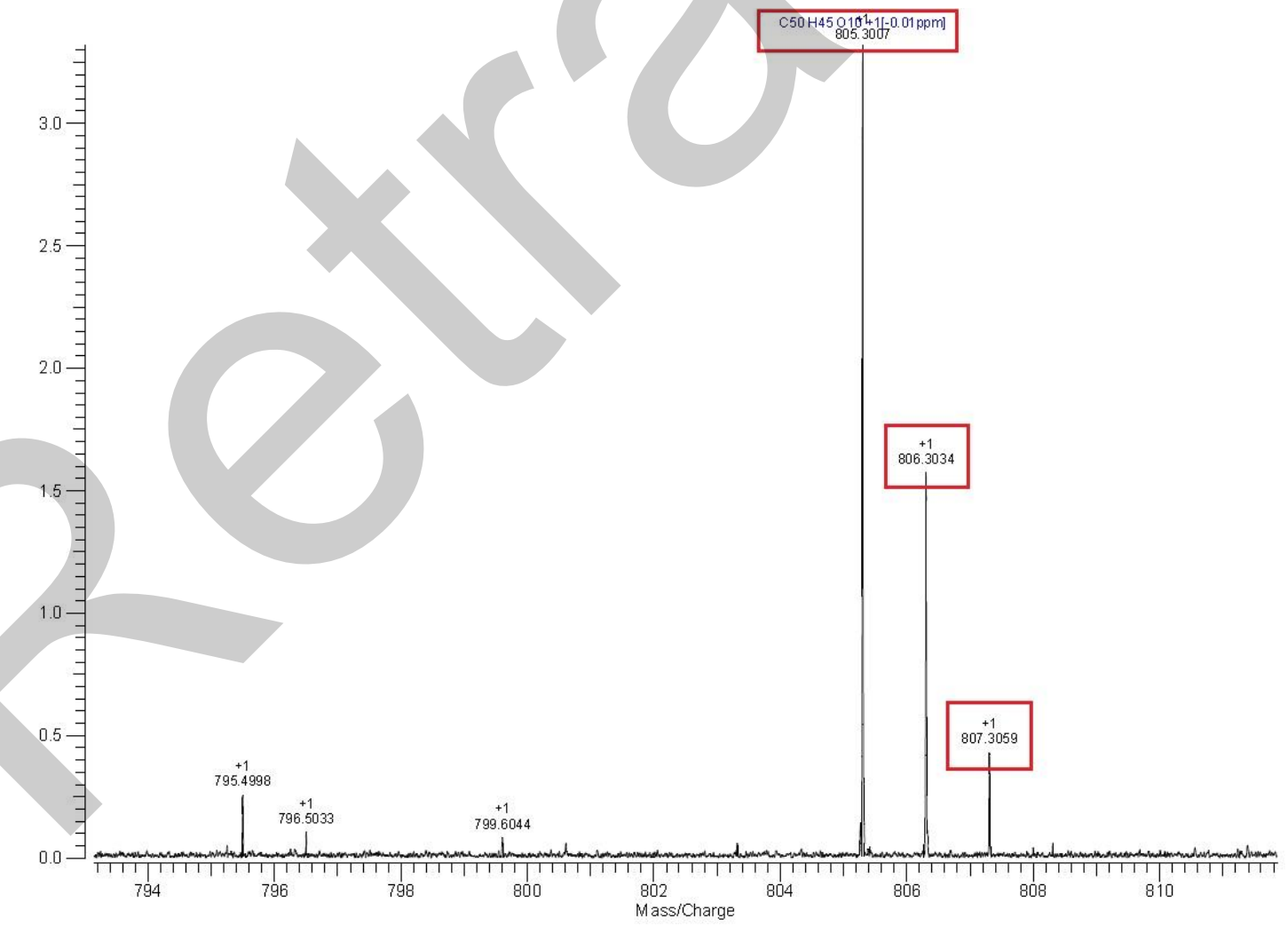


Fig. S11. ${ }^{1} \mathrm{H}$ NMR spectrum of $(\mathbf{1 + 2})$ in $\mathrm{CDCl}_{3}\left({ }^{1} \mathrm{H}: 400 \mathrm{MHz}\right)$

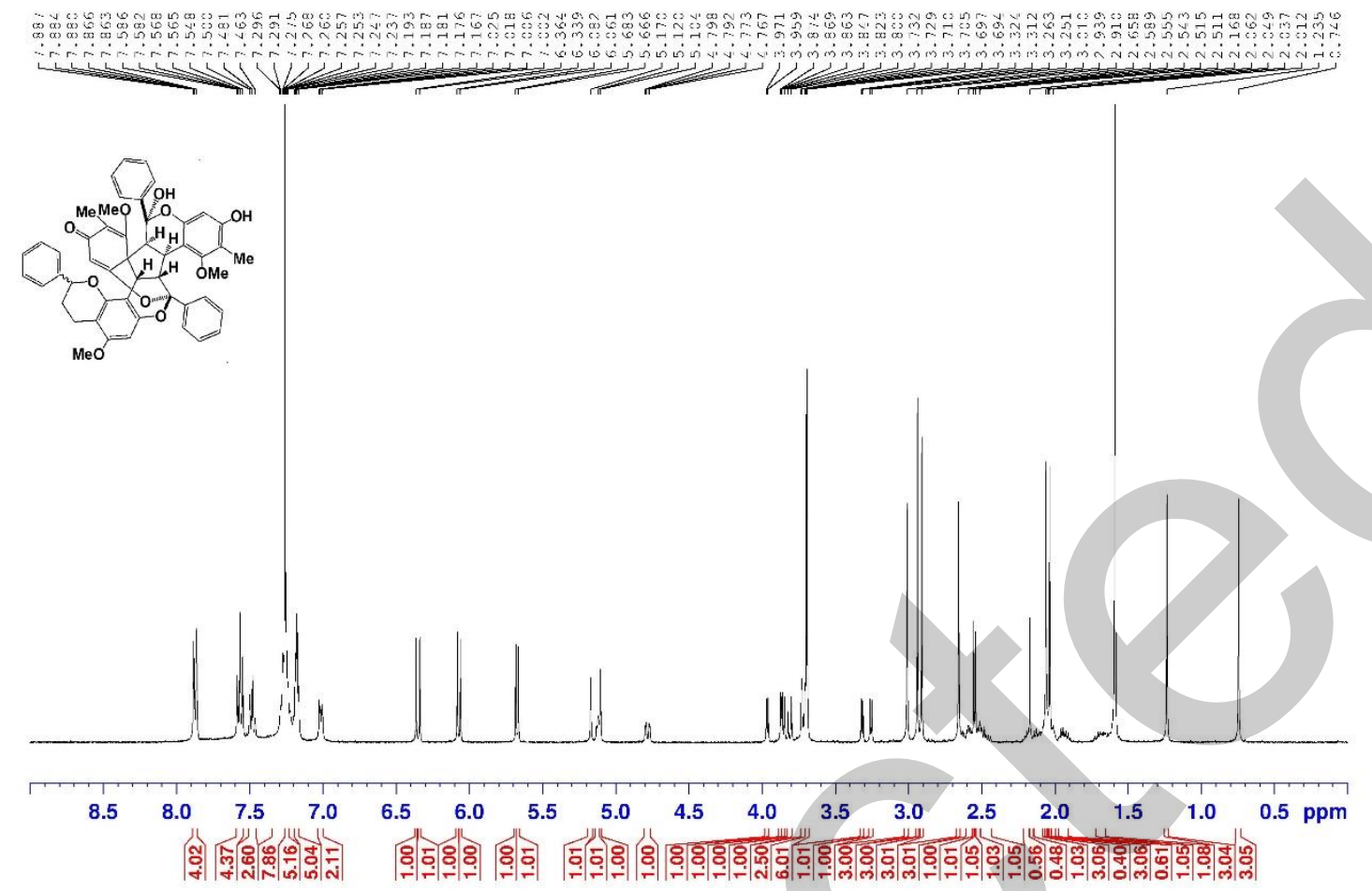

Fig. S12. ${ }^{13} \mathrm{C} \&$ DEPT-135 NMR spectrum of $(\mathbf{1 + 2})$ in $\mathrm{CDCl}_{3}\left({ }^{13} \mathrm{C}: 400 \mathrm{MHz}\right)$

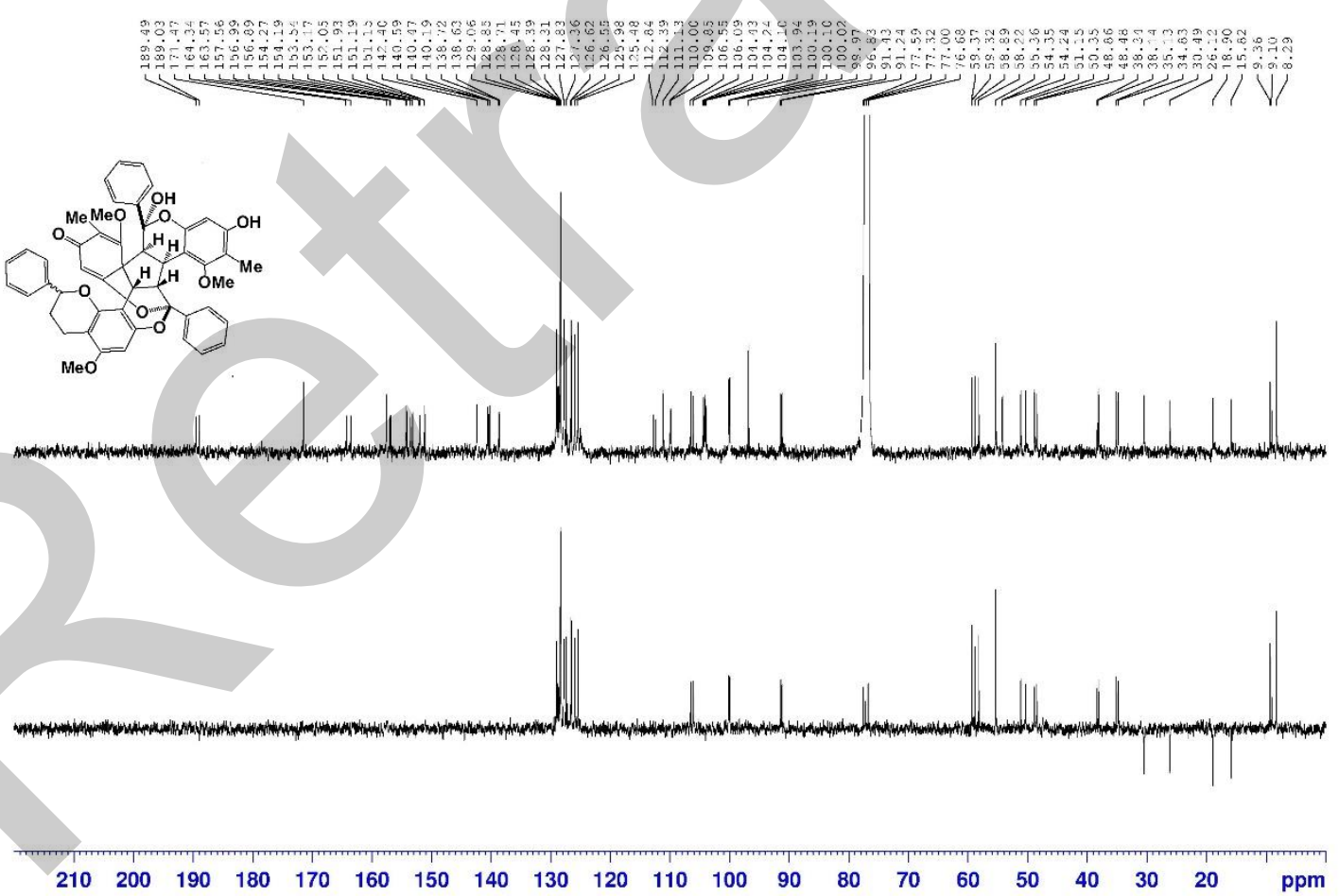


Fig. S13. ${ }^{1} \mathrm{H}$ NMR spectrum of 1 in $\mathrm{CDCl}_{3}\left({ }^{1} \mathrm{H}: 700 \mathrm{MHz}\right)$

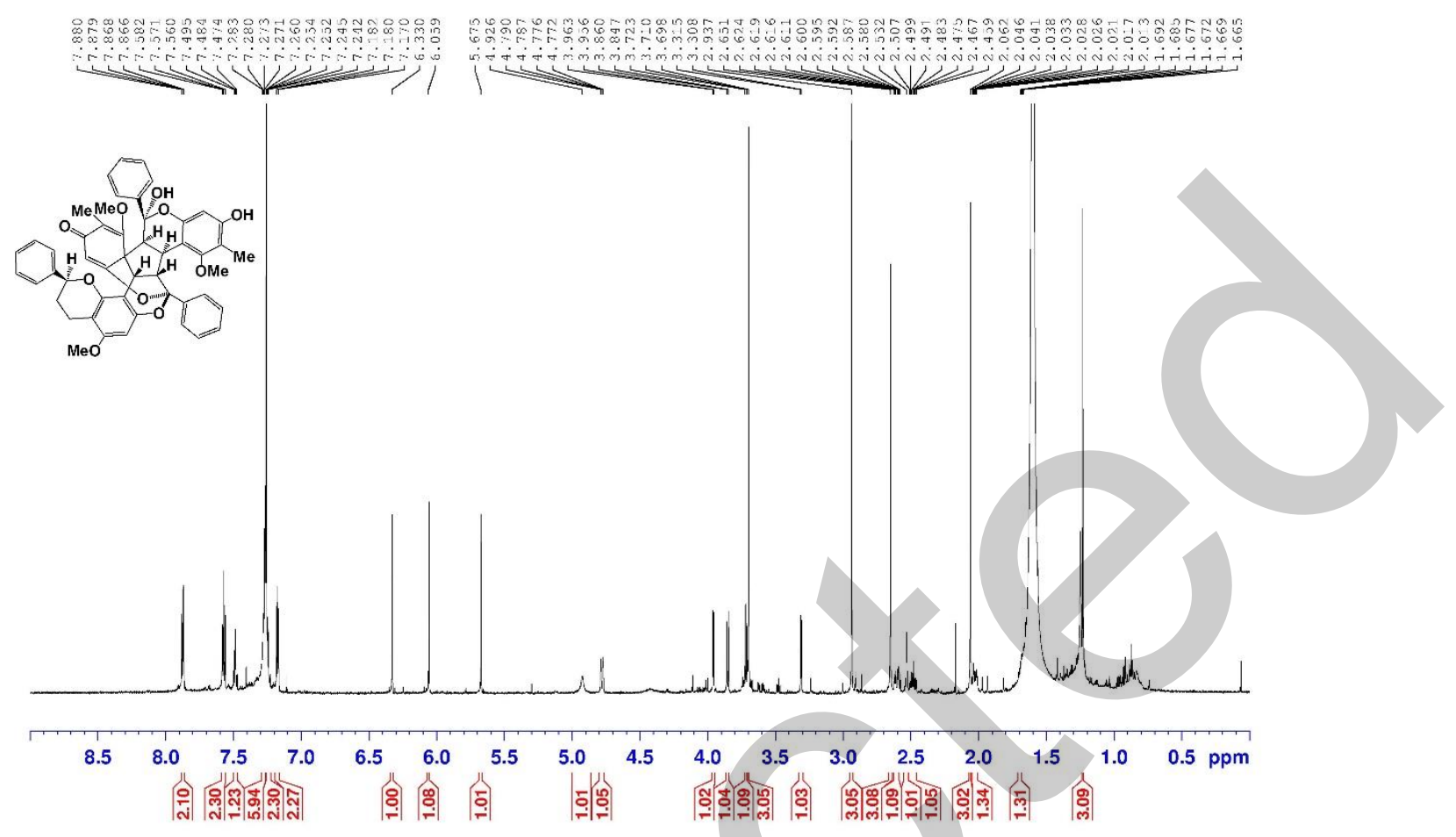

Fig. S13-1. ${ }^{1} \mathrm{H}$ NMR spectrum of 1 in $\mathrm{d}_{6}$-acetone

DDBC-57b18-cry_b Acetone-d6 2014/09/12 AVIII HD 700 293K

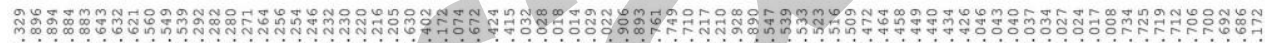

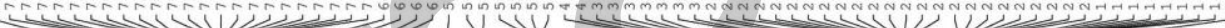

$\longrightarrow$ 
Fig. S14. ${ }^{13} \mathrm{C} \&$ DEPT-135 NMR spectrum of 1 in $\mathrm{d}_{6}$-Acetone $\left({ }^{13} \mathrm{C}: 700 \mathrm{MHz}\right)$

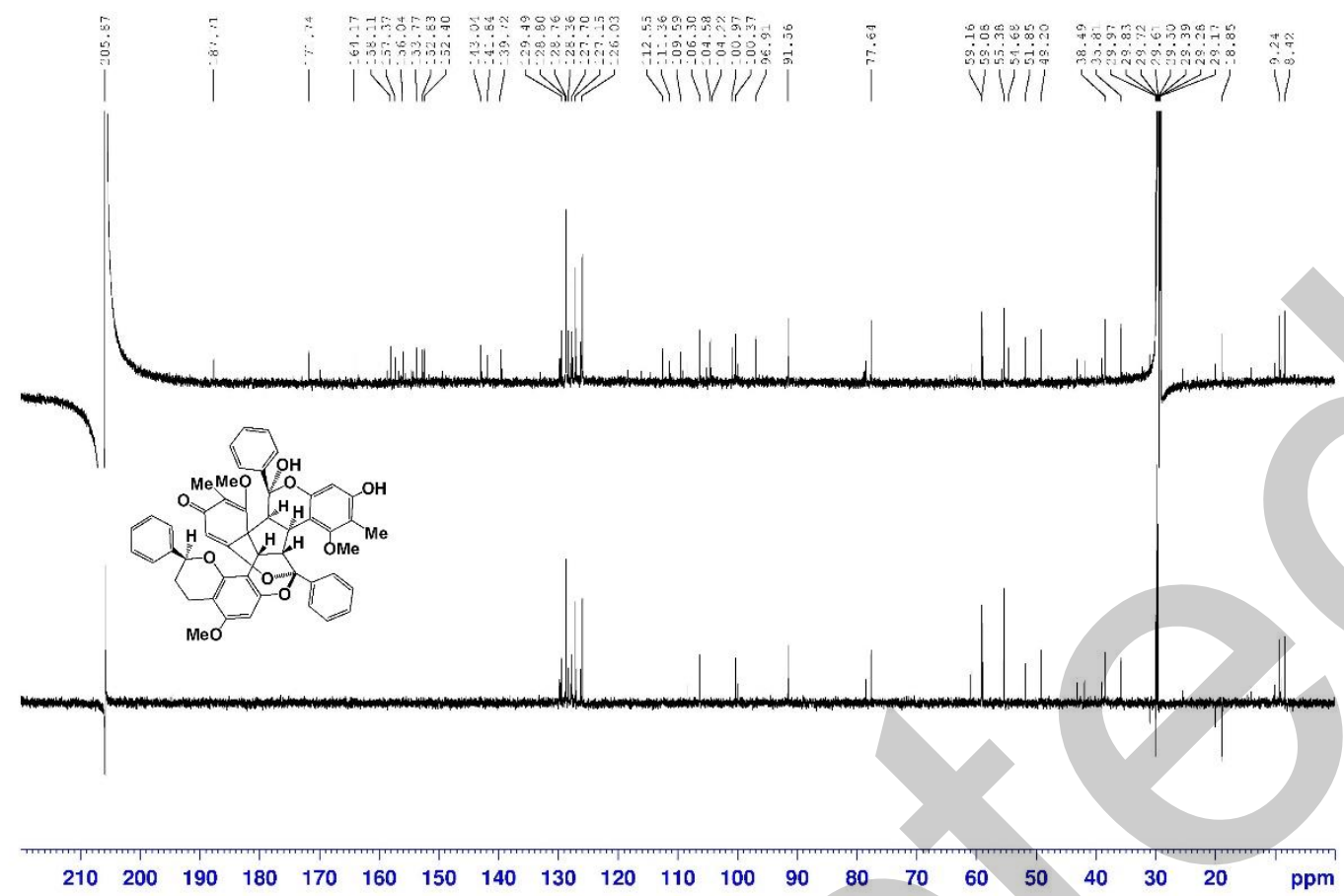

Fig. S15. ${ }^{1} \mathrm{H}-{ }^{1} \mathrm{H}$ COSY spectrum of 1 in $\mathrm{d}_{6}$-Acetone $\left({ }^{1} \mathrm{H}: 700 \mathrm{MHz}\right)$

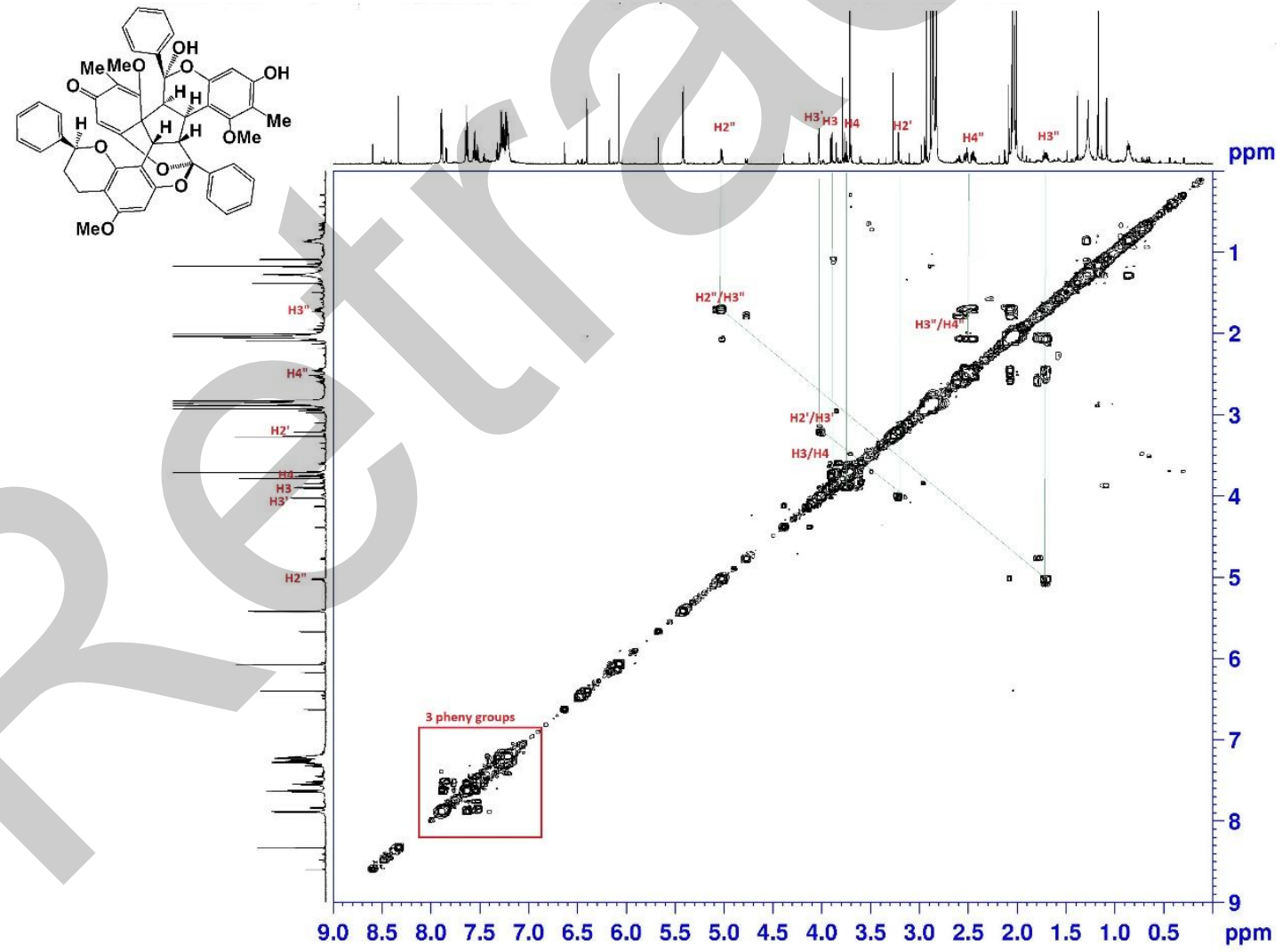


Fig. S16. HSQC spectrum of $\mathbf{1}$ in $\mathrm{d}_{6}$-Acetone $\left({ }^{1} \mathrm{H}-{ }^{13} \mathrm{C}\right.$ : $\left.700 \mathrm{MHz}\right)$

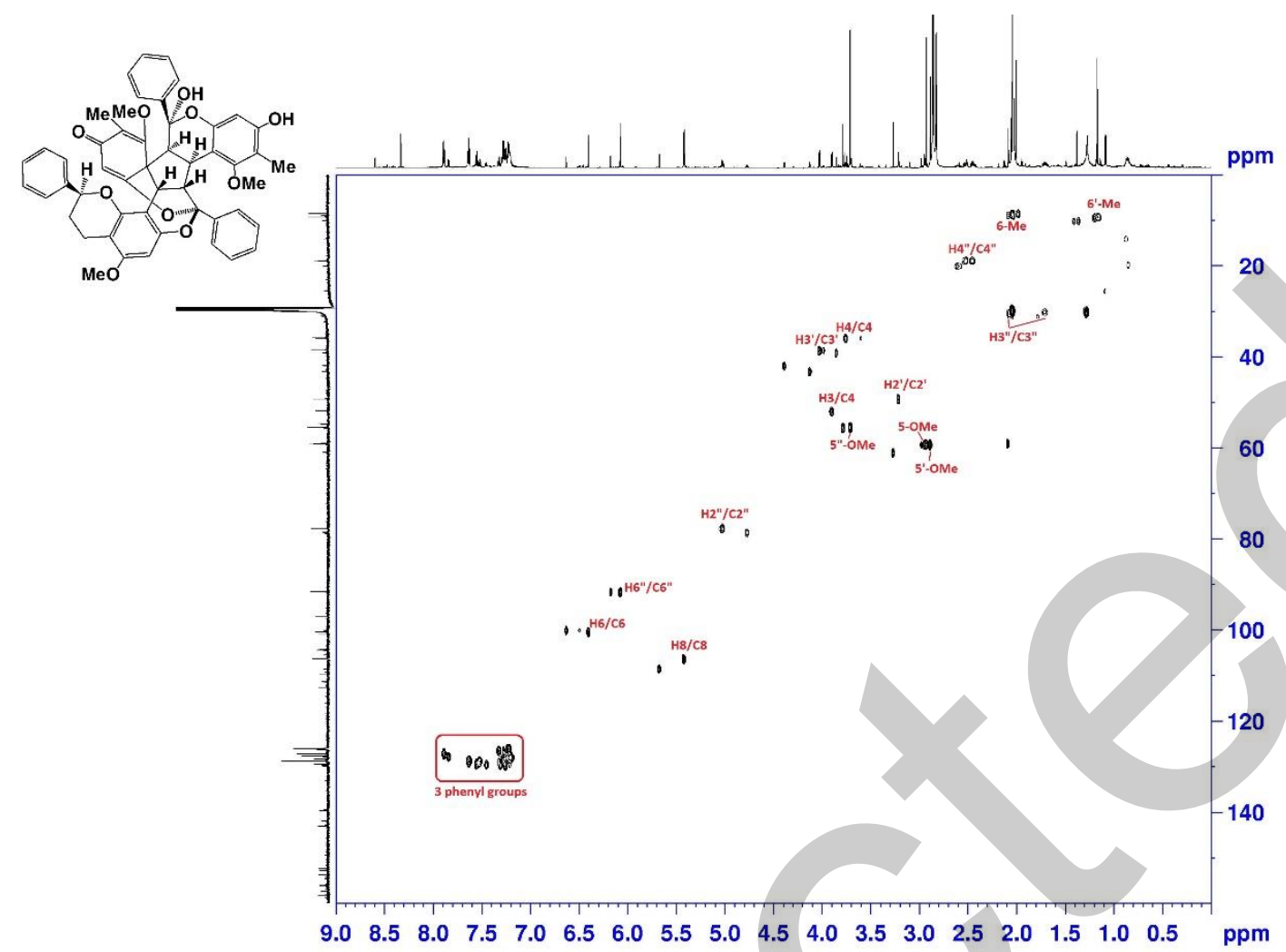

Fig. S17. HMBC spectrum of $\mathbf{1}$ in $\mathrm{d}_{6}$-Acetone $\left({ }^{1} \mathrm{H}-{ }^{13} \mathrm{C}: 700 \mathrm{MHz}\right)$

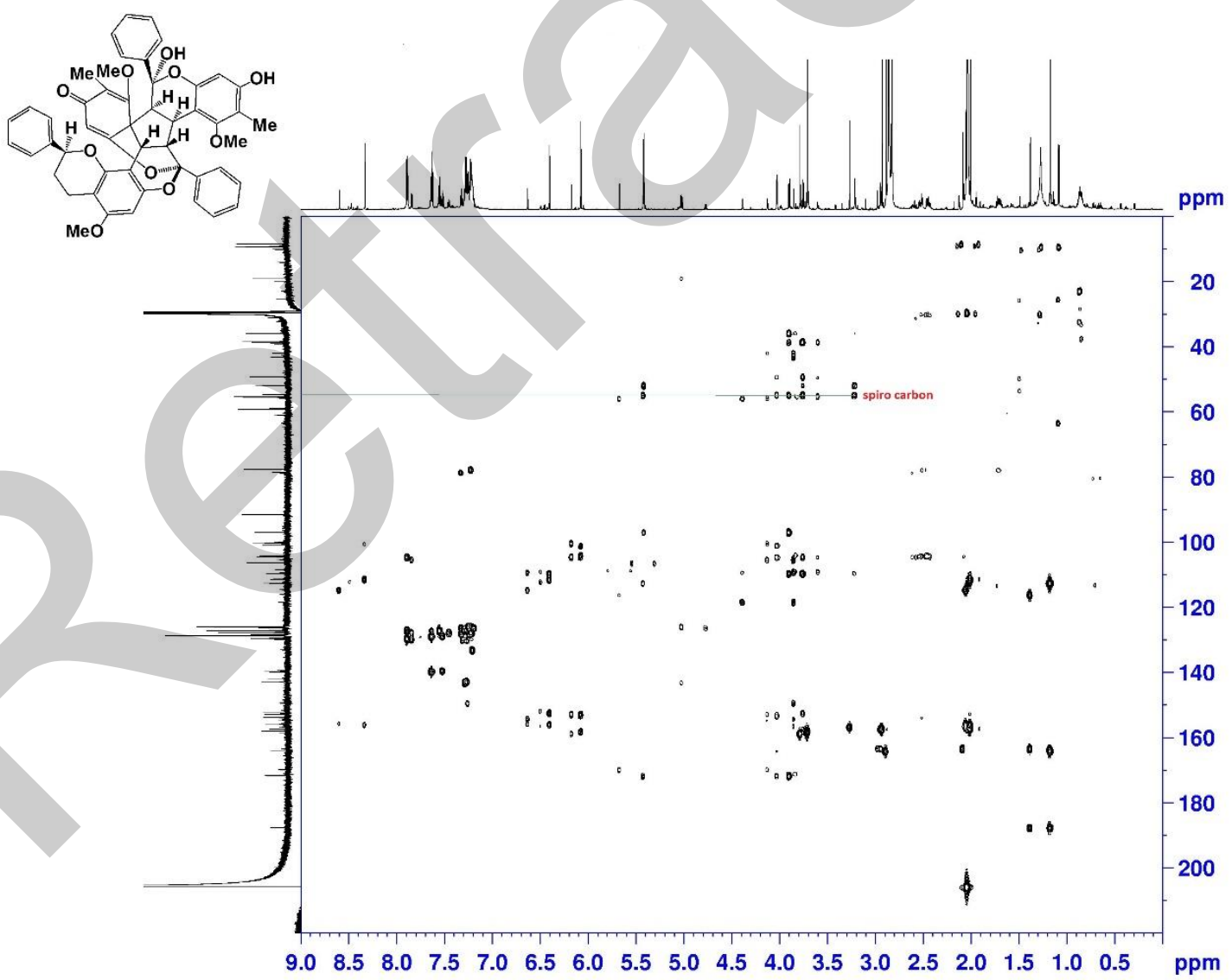


Fig. S18. Key NOESY spectrum of $\mathbf{1}$ in $\mathrm{CDCl}_{3} \quad\left({ }^{1} \mathrm{H}-{ }^{1} \mathrm{H}: \quad 700 \mathrm{MHz}\right)$

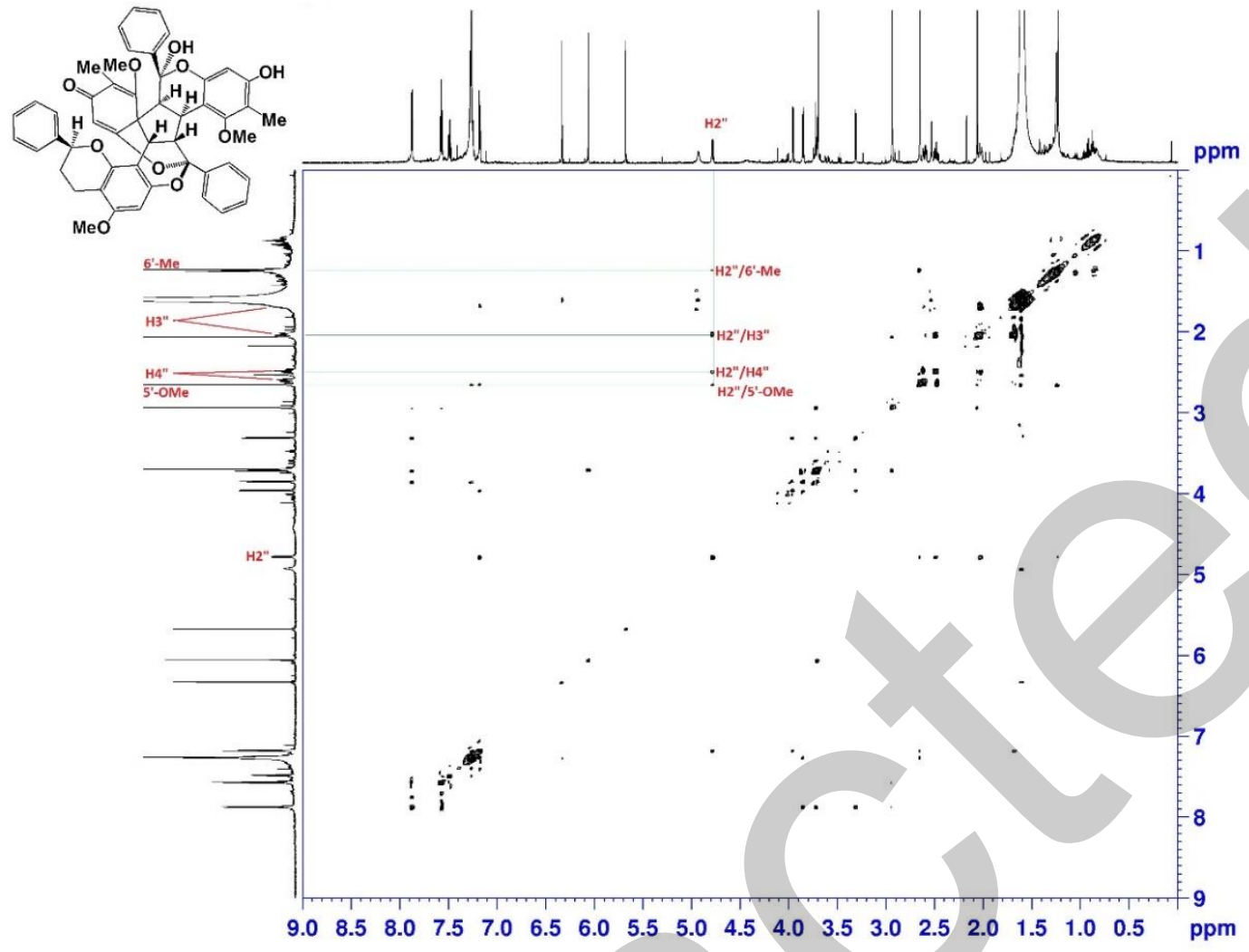

Fig. S19. ${ }^{1} \mathrm{H}$ NMR spectrum of 2 in $\mathrm{CDCl}_{3}\left({ }^{1} \mathrm{H}: 700 \mathrm{MHz}\right)$

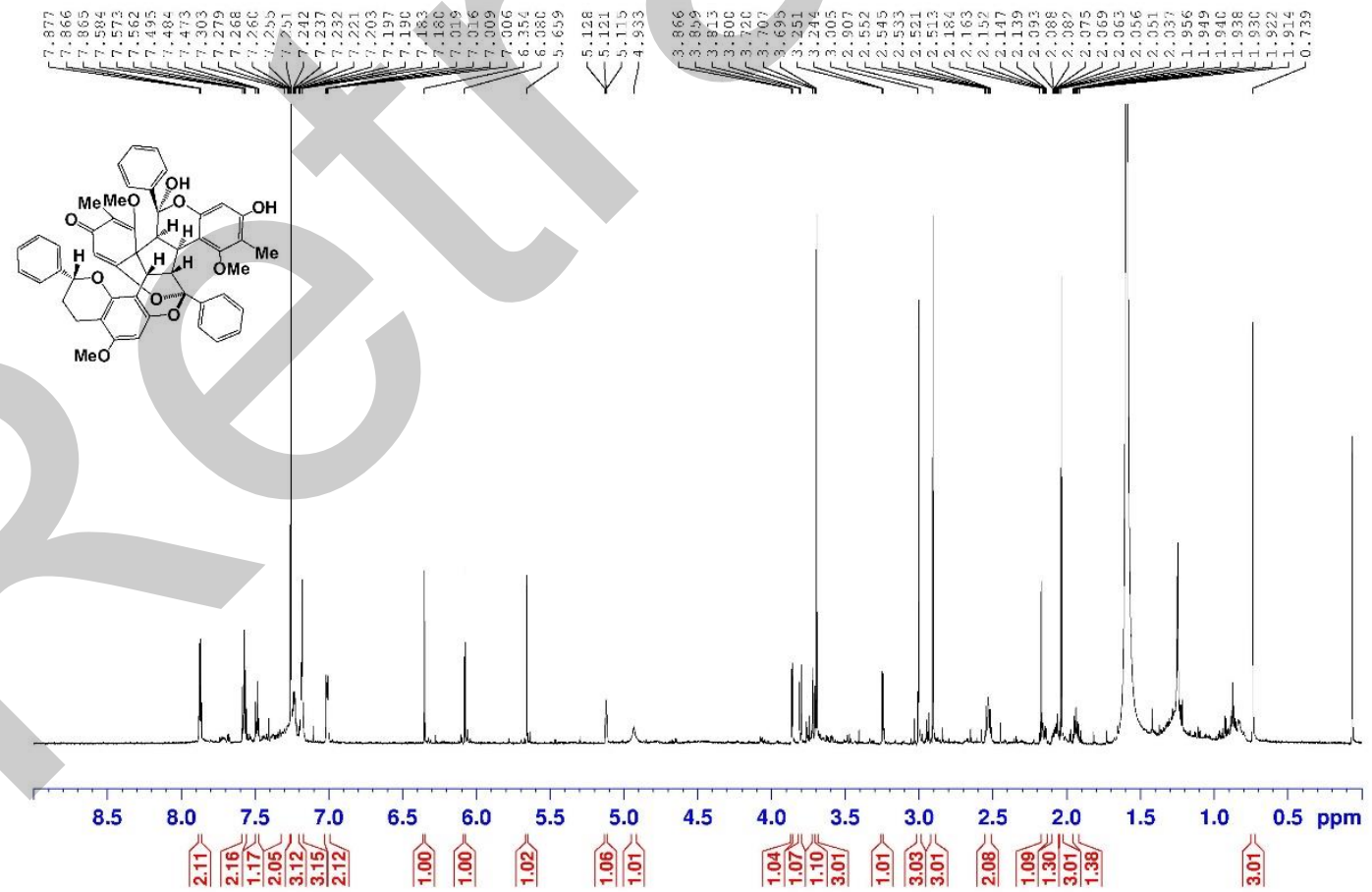


Fig. S19-1. ${ }^{1} \mathrm{H}$ NMR spectrum of 2 in $\mathrm{d}_{6}$-acetone $\left({ }^{1} \mathrm{H}: 700 \mathrm{MHz}\right)$.

DDBC-57b18-cry_a Acetone-d6 2014/09/12 AVIII HD 700 293K

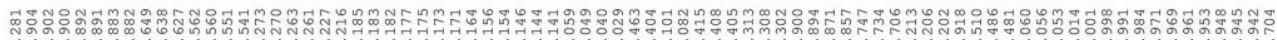

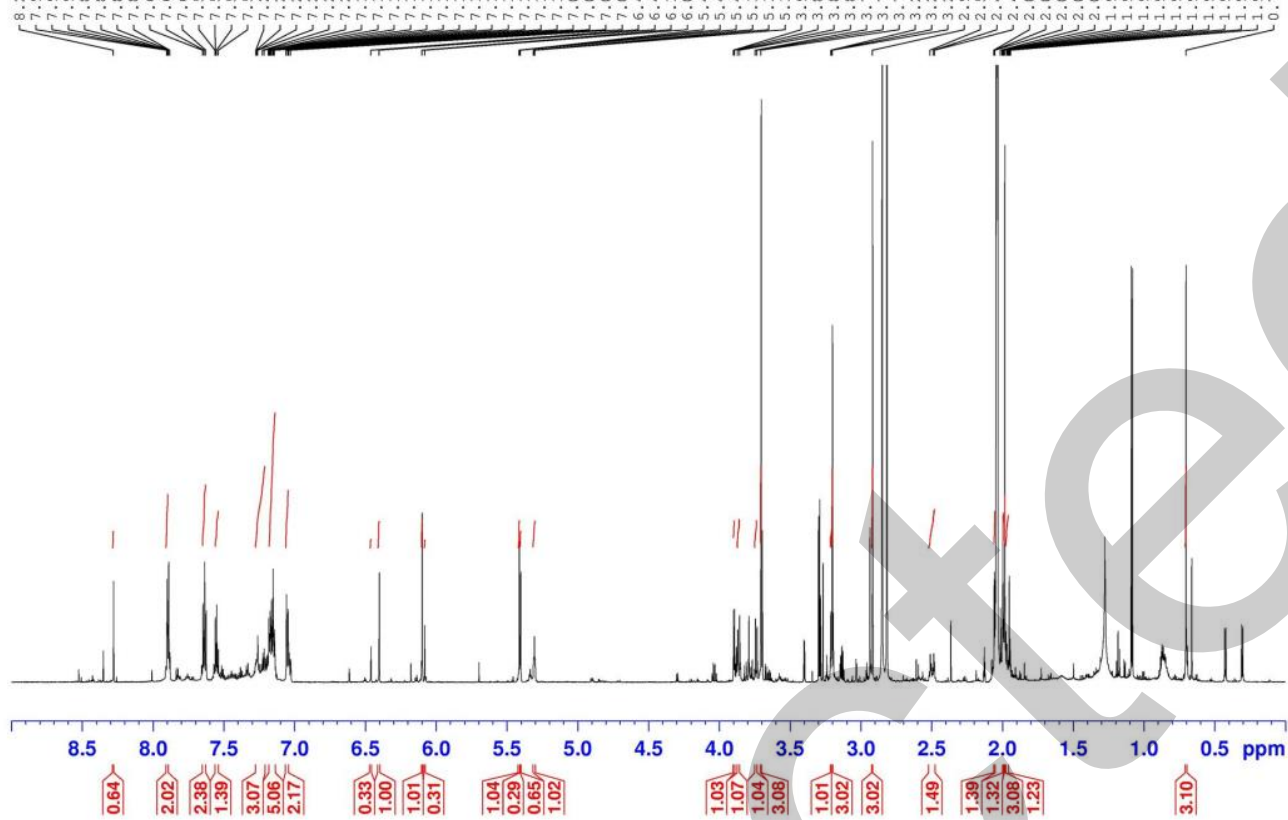

Fig. S20. ${ }^{13} \mathrm{C} \&$ DEPT-135 NMR spectrum of 2 in $\mathrm{d}_{6}$-Acetone $\left({ }^{13} \mathrm{C}: 700 \mathrm{MHz}\right)$ JDBC-57b18-cry_a Acetone-c.6 2014/09/11 AVIII HD 700 293K

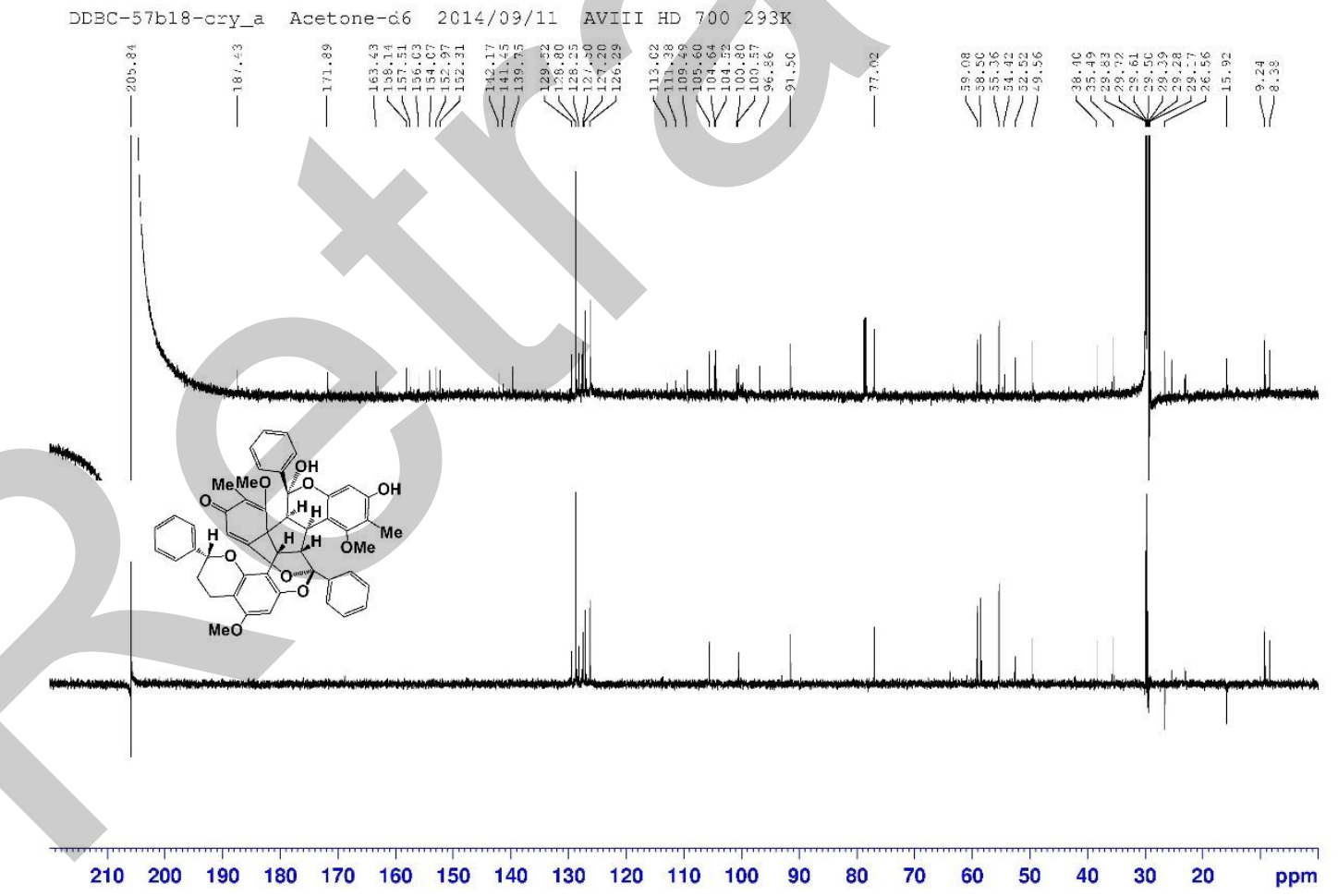


Fig. S21. ${ }^{1} \mathrm{H}-{ }^{1} \mathrm{H}$ COSY spectrum of 2 in $\mathrm{d}_{6}$-Acetone $\left({ }^{1} \mathrm{H}: 700 \mathrm{MHz}\right)$

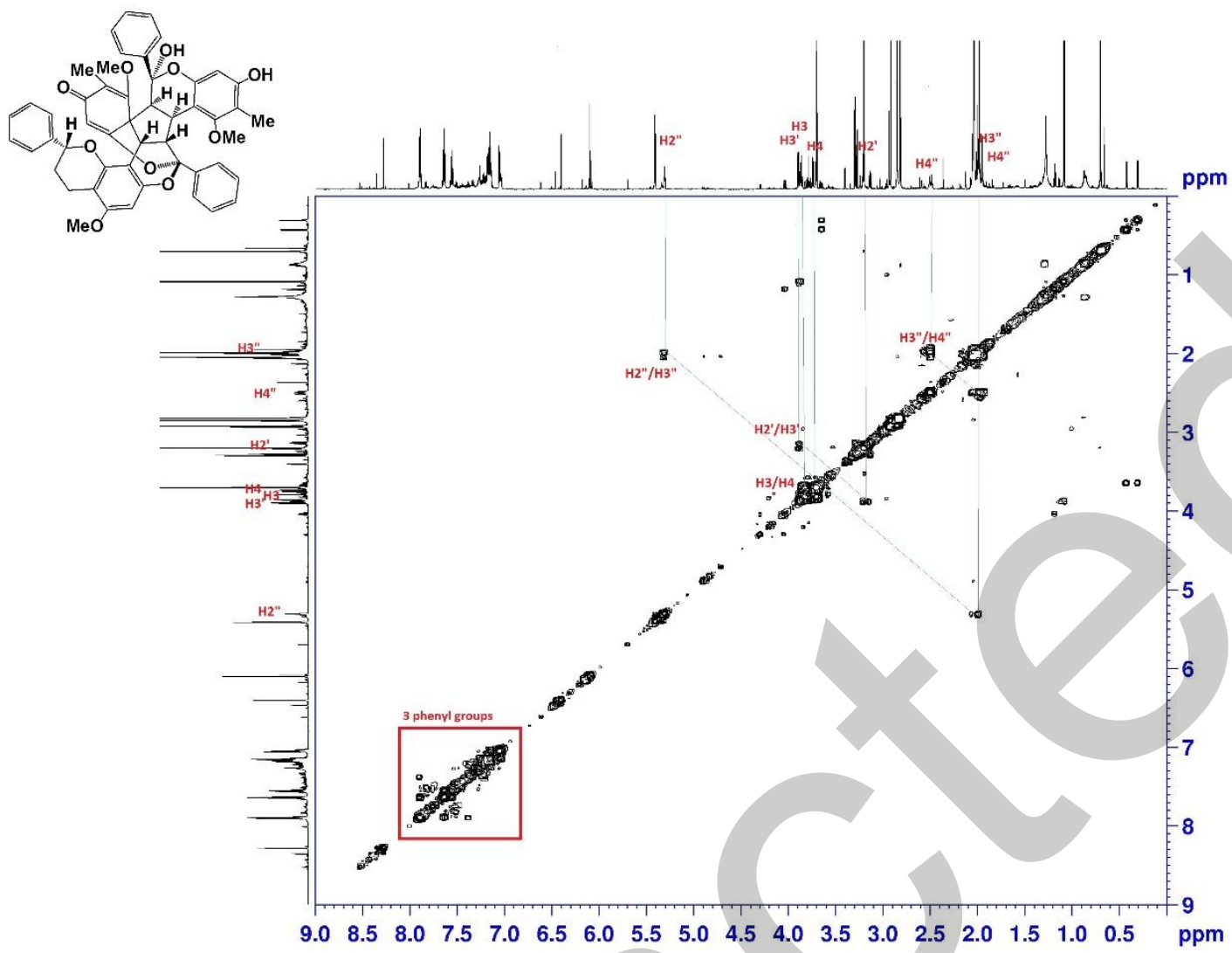

Fig. S22. HSQC spectrum of 2 in d6-Acetone $\left({ }^{1} \mathrm{H}-{ }^{13} \mathrm{C}: 700 \mathrm{MHz}\right)$

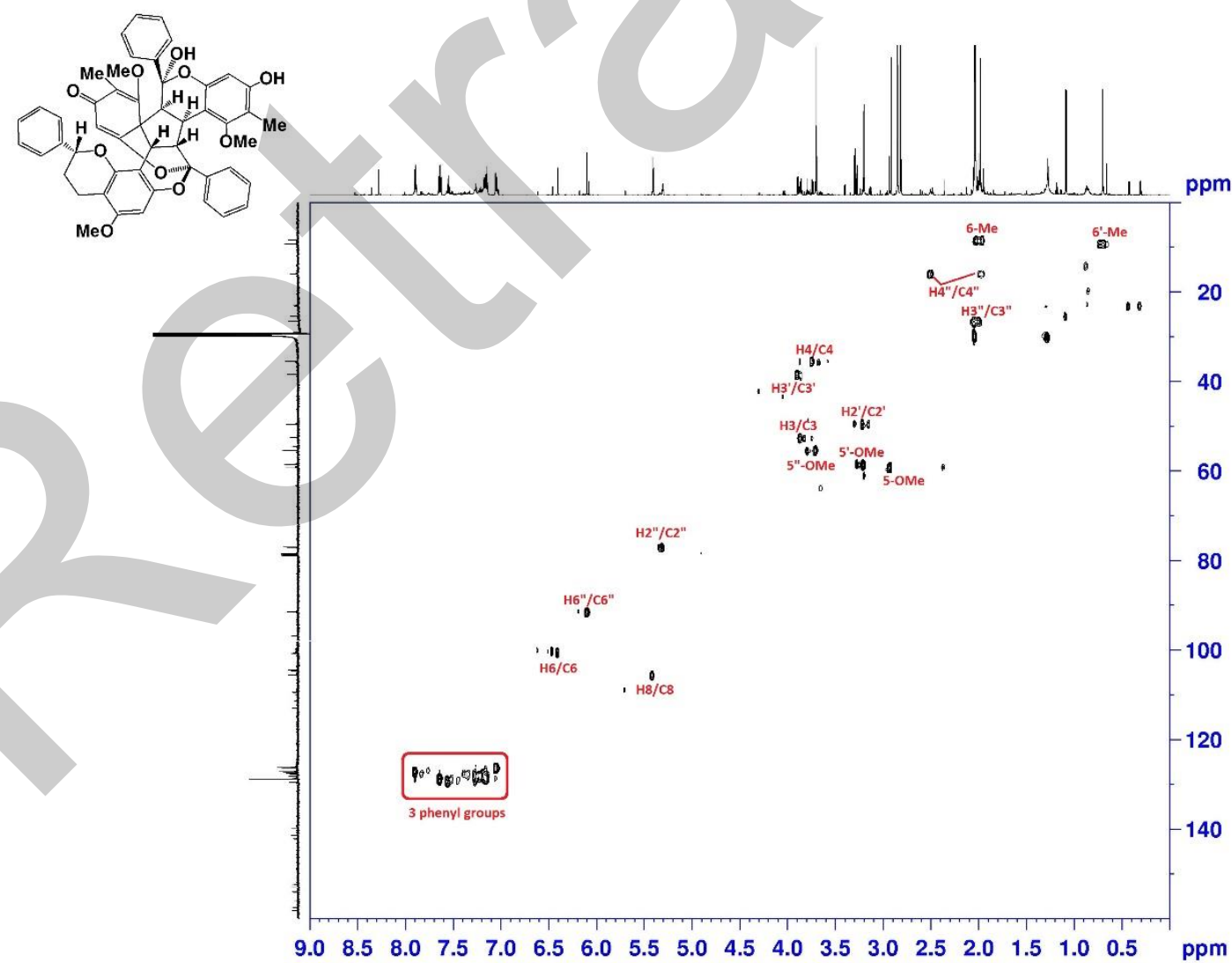


Fig. S23. HMBC spectrum of 2 in $\mathrm{d}_{6}$-Acetone $\left({ }^{1} \mathrm{H}^{13} \mathrm{C}: 700 \mathrm{MHz}\right)$

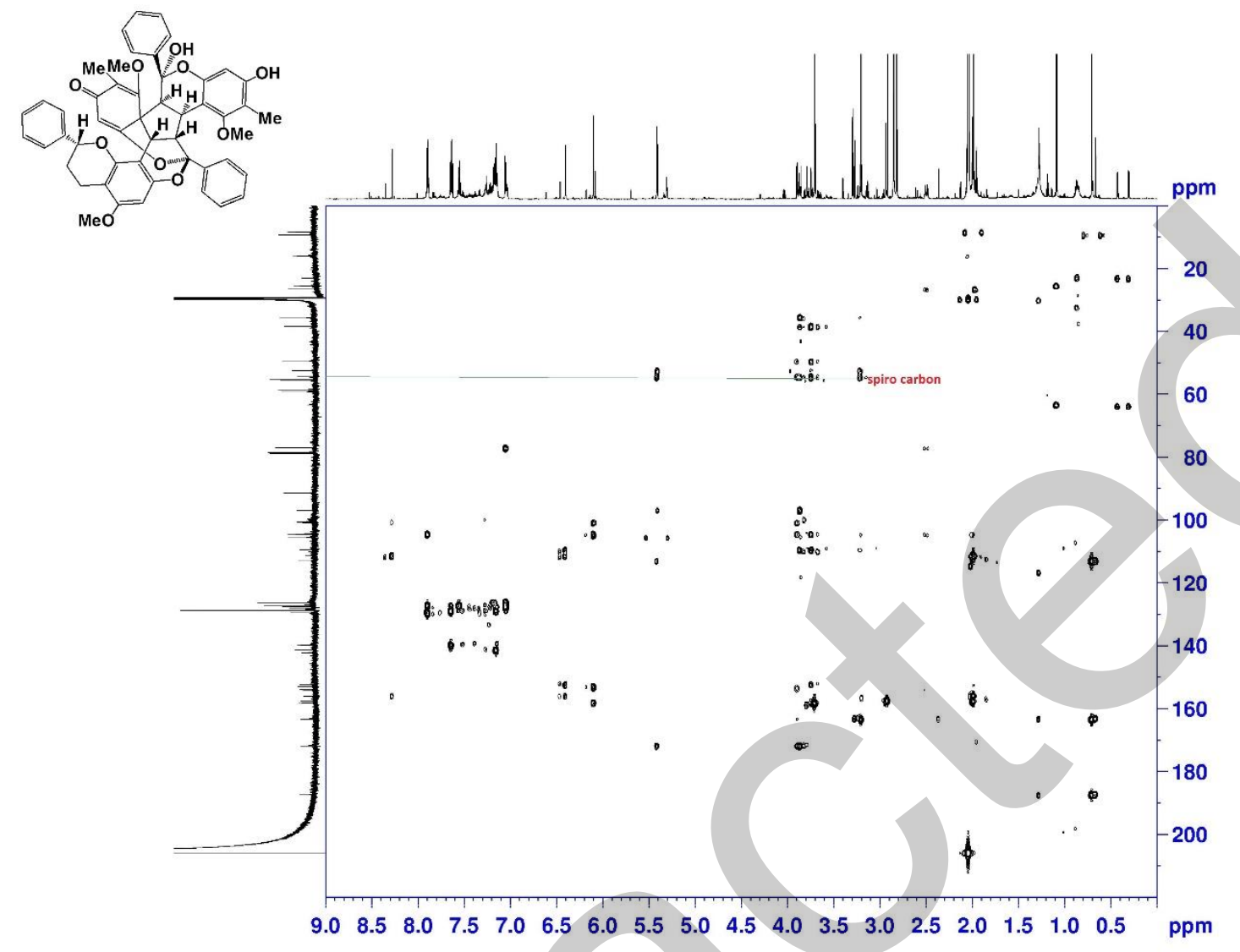

S24 
<smiles>[CH]1[C@@H]2C=C[C@@H]1C2</smiles> 
Fig. S25. H2"\&6'-Me had NOE on 3.4Å (A1, green); had no NOE on $6.3 \AA$ for A2 (red).
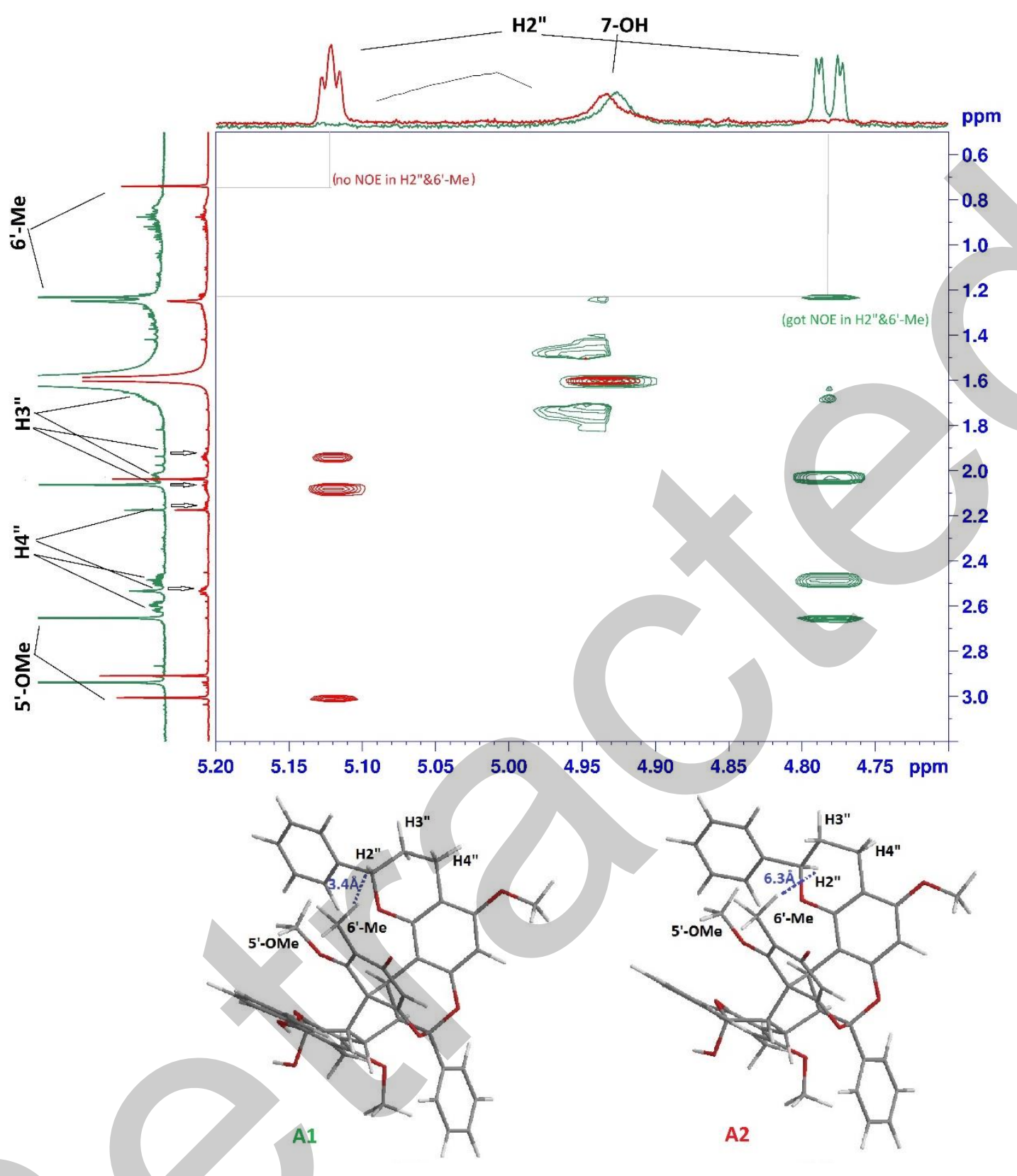

S26 
Fig. S26. A1 and A2 would transform to other compounds (quick-dry: collected and then concentrated soon after per inject by chiral-HPLC) shown in ${ }^{1} \mathrm{H}-\mathrm{NMR}$ in $\mathrm{d}_{6^{-}}$ acetone.
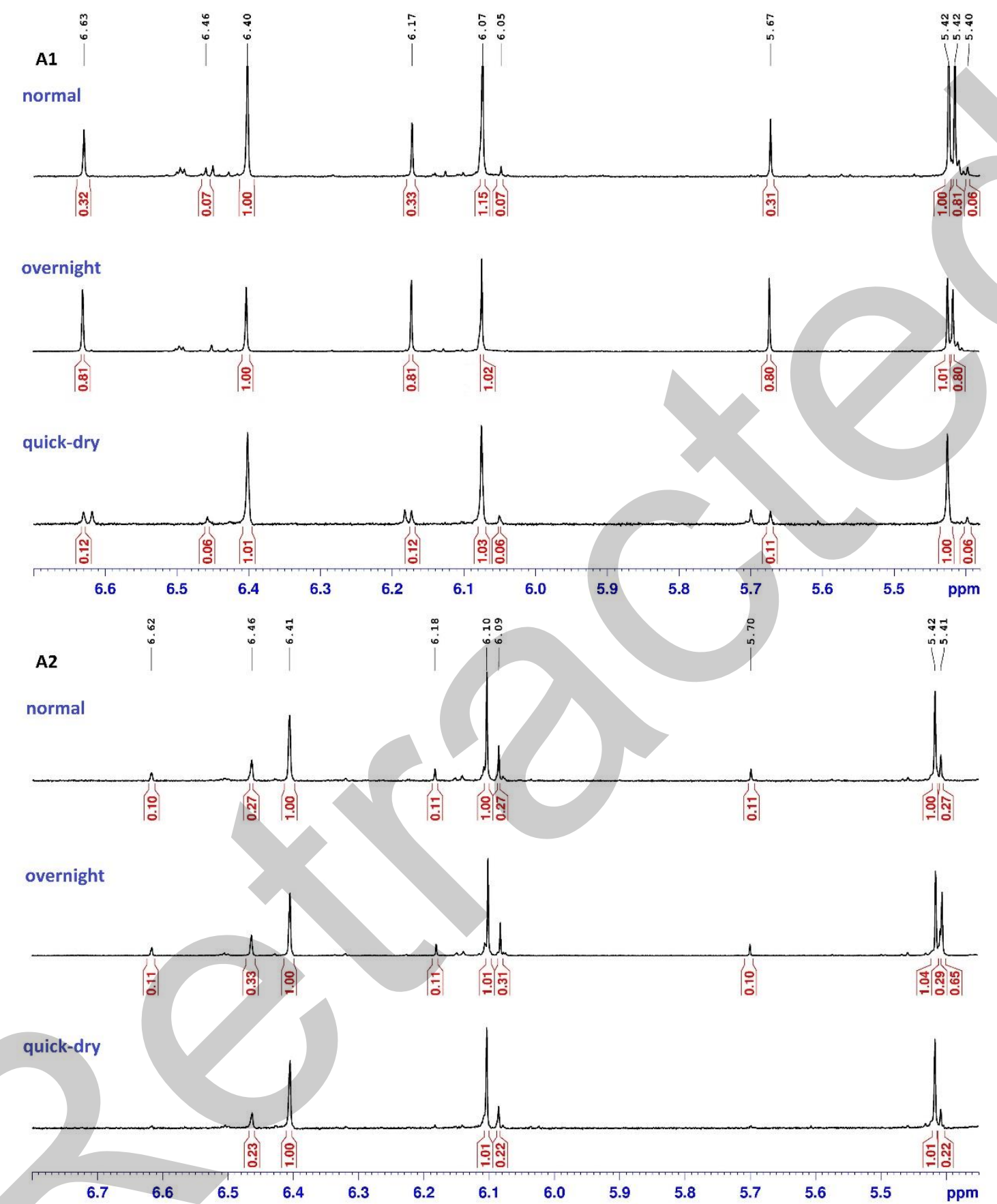
Fig. S27 Biosynthetic pathway of 1 and 2

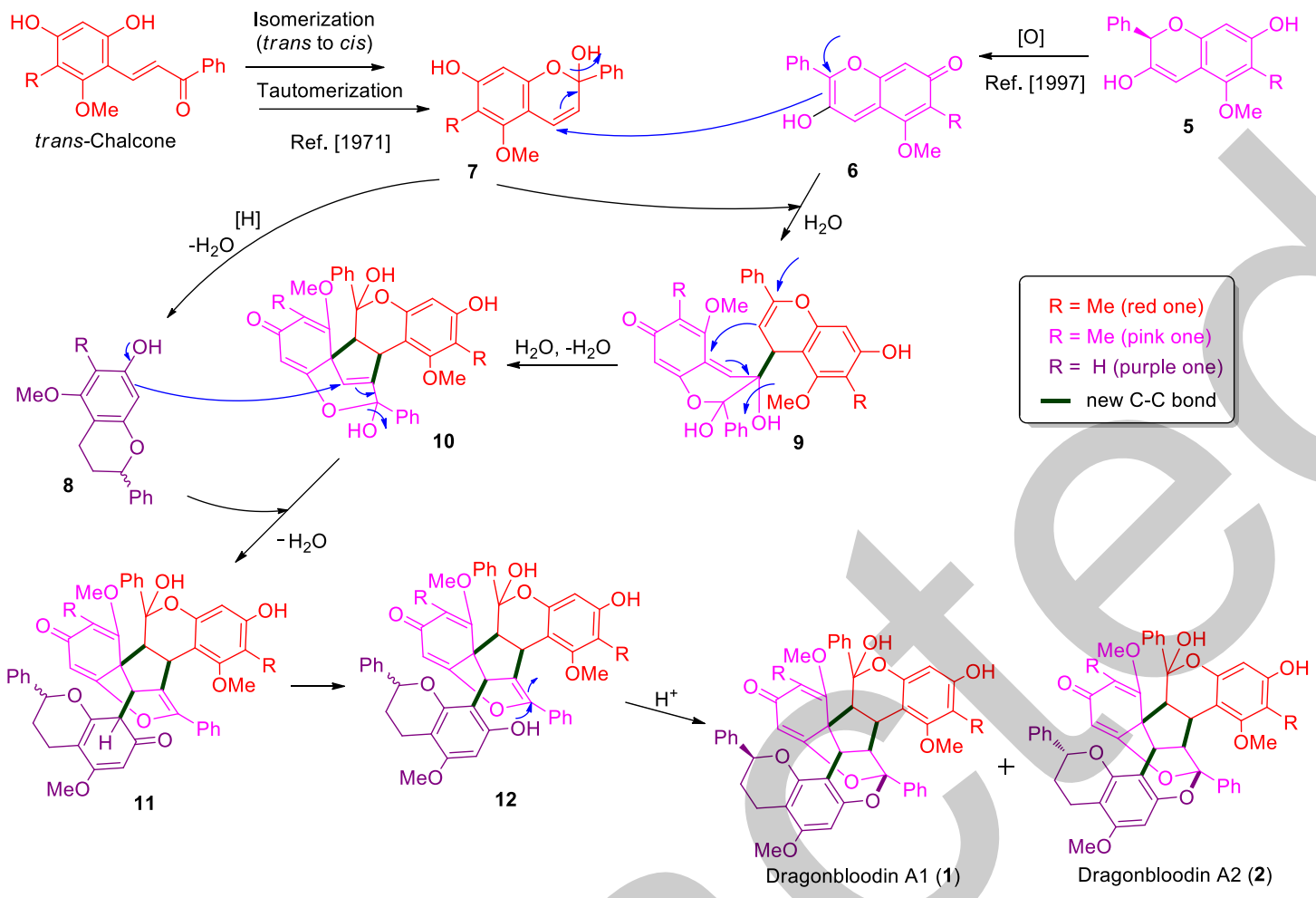

Fig. S28 Effects of dragonbloodin A1 (1) on the activities human neutrophil elastase in cell-free system. All data are expressed as the mean \pm S.E.M. $(n=3)$.

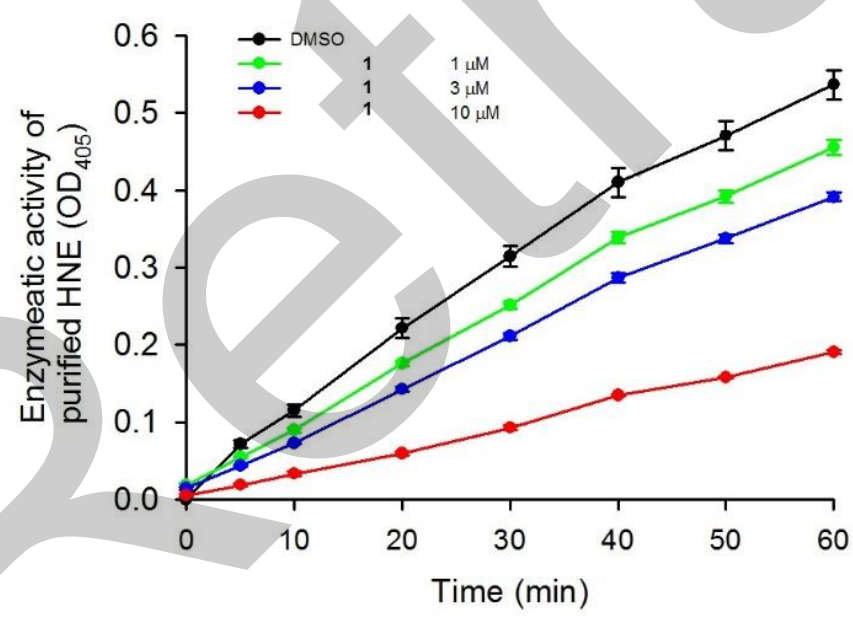


<smiles>C1C2C3CC2C13</smiles> 